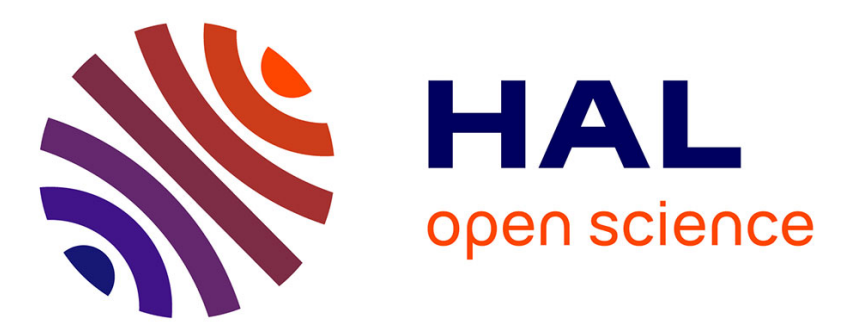

\title{
Stabilization of a class of underactuated vehicles with uncertain position measurements and application to visual servoing
}

\author{
Henry de Plinval, Pascal Morin, Philippe Mouyon
}

\section{- To cite this version:}

Henry de Plinval, Pascal Morin, Philippe Mouyon. Stabilization of a class of underactuated vehicles with uncertain position measurements and application to visual servoing. Automatica, 2017, 77, pp.155 - 169. 10.1016/j.automatica.2016.11.012 . hal-01475915

\section{HAL Id: hal-01475915 https://hal.sorbonne-universite.fr/hal-01475915}

Submitted on 24 Feb 2017

HAL is a multi-disciplinary open access archive for the deposit and dissemination of scientific research documents, whether they are published or not. The documents may come from teaching and research institutions in France or abroad, or from public or private research centers.
L'archive ouverte pluridisciplinaire HAL, est destinée au dépôt et à la diffusion de documents scientifiques de niveau recherche, publiés ou non, émanant des établissements d'enseignement et de recherche français ou étrangers, des laboratoires publics ou privés. 


\title{
Stabilization of a Class of Underactuated Vehicles with Uncertain Position Measurements and Application to Visual Servoing ${ }^{\underline{\lambda}}$
}

\author{
H. de Plinval ${ }^{\mathrm{a}}$, P. Morin ${ }^{\mathrm{b}}$, P. Mouyon ${ }^{\mathrm{a}}$, \\ ${ }^{a}$ ONERA - The French Aerospace Lab, Toulouse, France. Henry.de_Plinval@onera.fr, Philippe.Mouyon@onera.fr \\ ${ }^{b}$ ISIR, Sorbonne Universités, UPMC Univ. Paris 06, CNRS UMR 7222, Paris, France. Corresponding author. morin@isir.upmc.fr
}

\begin{abstract}
Stabilization of a class of underactuated vehicles with uncertain measurements of the position tracking error is addressed. Nonlinear feedback laws ensuring semi-global stability for a large class of uncertainties on these measurements are derived based on properties of saturated controls. Practical relevance of the proposed results is illustrated by two application examples for Vertical Take-Off and Landing aerial vehicles equipped with a mono-camera sensor: point stabilization in front of a planar target and visual way-points navigation based on interpolation of homography measures.
\end{abstract}

Keywords: Underactuated vehicle, aerial vehicle, stability, robustness, measurement uncertainties

\section{Introduction}

Underactuated vehicles have long been a source of inspiration for nonlinear control theory. Recent applications with aerial or underwater vehicles have renewed the interest on this topic. This study is motivated by applications with VTOL UAVs (i.e. Vertical Take-Off and Landing Unmanned Aerial Vehicles) but it is relevant to any underactuated vehicle that can be modeled as a rigid body with a body fixed thrust control force and full torque control (so-called "thrust propelled vehicles" Hua et al. (2009)). Stabilization of the vehicle's pose (i.e. position and orientation) is an important issue in this context. Large stability domains are needed for small vehicles due to their sensitivity to perturbations (wind, sea currents, etc), and several nonlinear control designs have been proposed to address this issue (see, e.g., Hauser et al. (1992); Isidori et al. (2003); Pflimlin et al. (2007); Hua et al. (2009)). Good robustness properties of the closed-loop system is at least as important in practice. Robustness to external perturbations (e.g. wind effects for aerial vehicles or currents for underwater vehicles) has been addressed in Pflimlin et al. (2007); Marconi and Naldi (2007); Aguiar and Pascoal (2007); Hua et al. (2009). Robustness to parameter uncertainties (mass, inertia, etc) has been considered e.g. in Aguiar and Pascoal (2007). Robustness to input disturbances has been considered in Aguiar et al. (2007). This paper concerns robustness w.r.t. (with respect to) uncertainties on the measurement model.

Motion capture systems provide high-quality pose measurements that can yield impressive performance for small aerial vehicles Lupashin et al. (2010); Mellinger et al. (2012). Alternatively, ground based passive visual markers have been used to

\footnotetext{
This work has been supported by the "Chaire d'excellence en Robotique RTE-UPMC" and the ANR SCAR Project.
}

estimate onboard the pose as in Masselli and Zell (2012). For most applications, however, motion capture systems or ground based localization systems cannot be used and localization must rely exclusively on embarked sensors. Good orientation estimates can be obtained with embarked IMUs (Inertial Measurement Units). Estimation of the vehicle's position/velocity is more challenging. GPS may be used to this purpose but it is not always available. Furthermore, in many applications (e.g., inspection) a measurement of the relative position of the vehicle w.r.t. its environment is needed, rather than an absolute position measurement (GPS-like). The former is best obtained from embarked exteroceptive sensors (cameras, lasers, etc). With such sensors, however, the relation between the output function (i.e. measurement) and the relative position error is seldom known precisely. Uncertainties may come from calibration errors, lack of depth information with mono-camera sensors, uncertainties on the environment structure, etc... This leads to the control problem addressed in this paper: Given a class of uncertainties on the position measurements, can one design feedback control laws that guarantee stability of the system for any position measurement in this class?

To our knowledge this problem has only been addressed in very specific cases, like when uncertainties reduce to a positive scale factor on the position vector Metni et al. (2004); Le Bras et al. (2010). The results here proposed address a much larger class of uncertainties. They make use of properties of saturated controls. There is a large litterature on this topic, especially for linear systems (see, e.g., Teel (1991); He et al. (2005)). In those works, it is assumed that the system's dynamics and state are perfectly known. Saturated controls have also been used for UAVs in order to ensure some type of robustness property, e.g., Teel (1996) and Lòpez-Araujo et al. (2010) for robustness w.r.t. input saturation, Marconi and Naldi (2007), Hua et al. (2009), and Lòpez-Araujo et al. (2010) for robustness w.r.t. unmodelled 
dynamics. We show that saturated controls can be instrumental in ensuring robustness properties w.r.t. measurement errors. More precisely, we propose nonlinear feedback laws that ensure semi-global stability of the pose tracking error for a large class of uncertainties on the position measurement. These results are reminiscent of Robust Stability results for linear systems (Doyle et al., 1992, Ch. 4), where the objective is to guarantee stability for a family of plants that satisfy some uncertainty bound w.r.t. a nominal system. Two application scenarios are addressed. In the first one we assume that velocity measurements are available, e.g., a GPS provides these measurements and an exteroceptive sensor (e.g., camera) provides position measurements w.r.t. the environment. In the second scenario no velocity measurement is available, i.e., "GPS-denied" environment. Note that position control of UAVs without velocity measurements has already been addressed Abdessameud and Tayebi (2010), but position was assumed to be perfectly known. Finally, let us remark that a preliminary version of this paper was presented in de Plinval et al. (2012).

The results here proposed are illustrated by visual servoing applications with mono-camera measurements. This type of application has been considered in several works (see, e.g., Pebrianti et al. (2010); Saripalli et al. (2003) and additional references in Section 6) usually with a camera pointing downward and observing a flat and horizontal ground, and under the assumption that altitude is measured independently. Our results provide stability guarantees for much more general application scenarios.

The paper is organized as follows. Background and problem statement are presented in Section 2. A preliminary result is provided in Section 3 for the fully actuated case. The main results on the underactuated case are provided in Sections 4 and 5: in Section 4 we assume that linear velocity measurements are available; in Section 5 such measurements are not available. Application to visual servoing is considered in Section 6, with simulation results given in Section 7. Proofs are given in the Appendix.

\section{Background and problem statement}

The $n \times n$ identity matrix is denoted as $I_{n}$. The transpose of a matrix $M$ is denoted as $M^{T}$. For any square matrix $M$, $M_{s}:=\frac{M+M^{T}}{2}$ and $M_{a}:=\frac{M-M^{T}}{2}$ respectively denote the symmetric and antisymmetric part of $M$. The maximum singular value of a matrix $M$ is denoted as $|M|$ and when $M$ is a matrix-valued time-function, $|M|_{s}:=\sup _{t}|M(t)|$. Given a matrix-valued timefunction $M: t \longmapsto M(t) \in \mathbb{R}^{n \times n}$ with $M(t) \geq 0 \forall t$, we define $|M|_{i}:=\sup _{\alpha I \leq M(t) \forall t} \alpha$. Note that $|M|_{i}=\left|M_{s}\right|_{i}$. Given a smooth function $f$ defined on an open set of $\mathbb{R}$, its derivative is denoted as $f^{\prime}$. Throughout the paper, AS, GAS, and LES stand for Asymptotically Stable, Globally Asymptotically Stable, and Locally Exponentially Stable respectively. CD stands for Convergence Domain.

Definition 1 Given $\delta:=\left[\delta_{m} ; \delta_{M}\right]$ with $0<\delta_{m}<\delta_{M}$, sat s $_{\delta}$ : $\mathbb{R}^{n} \longrightarrow \mathbb{R}^{n}$ is called a saturation function if: i) There exists a class $C^{1}$ function $\mathrm{s}_{\delta}: \mathbb{R}^{+} \longrightarrow \mathbb{R}^{+}$such that $\operatorname{sat}_{\delta}(x)=\mathrm{s}_{\delta}\left(|x|^{2}\right) x$ for all $x \in \mathbb{R}^{n}$;

ii) The function defined on $\mathbb{R}^{+}$by $\tau \longmapsto \mathrm{s}_{\delta}\left(\tau^{2}\right) \tau$ is nondecreasing, upper-bounded by $\delta_{M}$, and is equal to the identity function on $\left[0, \delta_{m}\right]$;

iii) $\mathrm{s}_{\delta}^{\prime}(\tau) \leq 0$ for all $\tau$.

From $i$ ) $\mathrm{sat}_{\delta}$ is fully defined from the associated function $\mathrm{s}_{\delta}$. From $i$ )-ii) saturation functions in the sense of Def. 1 inherit the classical properties of a saturation function: sat $_{\delta}$ is upperbounded in norm by $\delta_{M}$ and $\operatorname{sat}_{\delta}(x)=x$ for $|x| \leq \delta_{m}$ (because $\mathrm{s}_{\delta}\left(\tau^{2}\right) \tau=\tau$ for $\left.\tau \in\left[0, \delta_{m}\right]\right)$. Also,

$$
\begin{aligned}
& \mathrm{s}_{\delta}(\tau) \leq 1, \forall \tau \in \mathbb{R}^{+} \\
& \tau \mathrm{s}_{\delta}(\tau) \longrightarrow+\infty \quad \text { when } \quad \tau \longrightarrow+\infty
\end{aligned}
$$

where the first relation follows from ii) and iii) and the second relation from $i i)$. Then, ii) implies that the derivative of the function $\tau \longmapsto \mathrm{s}_{\delta}\left(\tau^{2}\right) \tau$ is non-negative. This property and iii) imply that $2 \tau\left|\mathrm{s}_{\delta}^{\prime}(\tau)\right| \leq \mathrm{s}_{\delta}(\tau) \forall \tau \in \mathbb{R}^{+}$. Thus, from (1),

$$
C_{\delta}:=\sup _{\tau \in \mathbb{R}^{+}}\left(\mathrm{s}_{\delta}(\tau)+2 \tau\left|\mathrm{s}_{\delta}^{\prime}(\tau)\right|\right) \leq 2<+\infty
$$

An example of a function $\mathrm{s}_{\delta}$ is given by

$$
\mathrm{s}_{\delta}(\tau):=\left\{\begin{array}{l}
1 \text { if } \tau \leq \delta_{m}^{2} \\
\frac{\delta_{M}}{\sqrt{\tau}}-\frac{\left(\delta_{M}-\delta_{m}\right)^{2}}{\sqrt{\tau}\left(\sqrt{\tau}+\delta_{M}-2 \delta_{m}\right)} \text { if } \tau>\delta_{m}^{2}
\end{array}\right.
$$

\subsection{Dynamics of thrust-propelled underactuated vehicles}

We focus on the class of so-called "thrust-propelled underactuated vehicles" Hua et al. (2009), i.e., rigid bodies moving in $3 D$-space under the action of one body-fixed force control and full torque control. This class contains most VTOL UAVs (quadrotors, ducted-fans, etc). The dynamics of these systems is described by the following equations, expressed in a "NorthEast-Down" (NED) frame:

$$
\left\{\begin{array}{l}
\ddot{p}=-u R e_{3}+g e_{3} \\
\dot{R}=R S(\omega) \\
J \dot{\omega}=J \omega \times \omega+\Gamma
\end{array}\right.
$$

with $p$ the position vector of the vehicle's center of mass, expressed in a reference (inertial) frame, $R$ the rotation matrix from the body frame to the reference frame, $\omega$ the angular velocity vector expressed in the body frame, $S($.) the matrix-valued function associated with the cross product, i.e. $S(x) y=x \times$ $y, \forall x, y \in \mathbb{R}^{3}, u$ the normalized thrust input, i.e. $u=\frac{T}{m}$ where $m$ is the mass and $T$ the thrust input, $e_{3}=(0,0,1)^{T}, J$ the inertia matrix, $\Gamma$ the torque vector, and $g$ the gravity constant. In this paper we mainly focus on the system

$$
\left\{\begin{array}{l}
\ddot{p}=-u R e_{3}+g e_{3} \\
\dot{R}=R S(\omega)
\end{array}\right.
$$

with $u$ and $\omega$ as control inputs, i.e., considering $\omega$ instead of $\Gamma$ as orientation control. Extension of the paper's results to System (4) is discussed in Section 4. 


\subsection{Problem statement}

The main objective of this paper is to investigate the stabilization of $p$ to a reference trajectory $p_{r}$ from some relative position measurements of the following form:

$$
\tilde{\sigma}(t):=R(t)^{T} M(t) \tilde{p}(t)
$$

with $M(t)$ an unknown matrix and $\tilde{p}:=p-p_{r}$ the position error. In other words $\tilde{\sigma}$ provides information on the position error in body frame and $M(t)$ accounts for measurements uncertainties. Measurements $\tilde{\sigma}$ are typically obtained from embarked exteroceptive sensors (cameras, lasers, etc). Examples are provided in Section 6. Due to the system's underactuation, stabilization of $p$ to $p_{r}$ fixes two degrees of freedom on the vehicle's orientation. There remains a degree of freedom (yaw angle). Its control is not addressed here since it does not affect the position control.

Let us introduce some assumptions. The first one is made throughout the paper. The other ones concern particular cases. A1: $M(t)>0 \forall t,|M|_{i}>0,|M|_{s}<+\infty,|\dot{M}|_{s}<+\infty$, and there exists a constant scalar $\left|\dot{p}_{r}\right|_{s}$ such that:

$$
\forall t, \quad\left|\dot{p}_{r}(t)\right| \leq\left|\dot{p}_{r}\right|_{s}
$$

A2: $M$ is a constant function.

A3: $\dddot{p}_{r}=0$ and $\left|\ddot{p}_{r}\right|<g$.

A4: $p_{r}$ is a constant vector and $M_{s}$ and $M_{a}$ commute.

\section{A preliminary result}

Before addressing the control of underactuated systems we consider a fully actuated system modelled by:

$$
\ddot{p}=-R u
$$

where $u \in \mathbb{R}^{3}$ denotes the body-fixed thrust input and $R$ satisfies the third kinematic relation in (5). We assume that the following measurements are available:

$$
\tilde{\sigma}, v:=R^{T} \dot{p}
$$

with $\tilde{\sigma}$ defined by (6) and $v$ the linear velocity in body frame. A reference trajectory is defined by:

$$
p_{r}, v_{r}:=R^{T} \dot{p}_{r}, a_{r}:=R^{T} \ddot{p}_{r}
$$

and the associated tracking error by:

$$
\tilde{p}:=p-p_{r}, \tilde{v}:=v-v_{r}
$$

Proposition 1 Let sat $_{\delta}, \overline{\text { sat }}_{\bar{\delta}}$ denote two $\mathbb{R}^{3}$-valued saturation functions with associated functions $\mathrm{s}_{\delta}, \overline{\mathrm{s}}_{\bar{\delta}}$. Consider control gains $k_{1}, k_{2}>0$ such that

$$
\left\{\begin{array}{l}
k_{2}^{2}|M|_{i}>k_{1}\left|M_{a}\right|_{s} \max \left\{C_{\delta}|M|_{s},\left|M_{a}\right|_{s}\right\} \\
k_{2} \bar{\delta}_{m}>k_{1} \delta_{M}
\end{array}\right.
$$

and define the control law

$$
u:=k_{1} \operatorname{sat}_{\delta}(\tilde{\sigma})+k_{2} \overline{\operatorname{sat}}_{\bar{\delta}}(\tilde{v})-a_{r}
$$

Then,

i) if $\mathbf{A} 1$ and $\mathbf{A} 2$ hold, then $(p, \dot{p})=\left(p_{r}, \dot{p}_{r}\right)$ is a (uniformly) GAS and LES equilibrium trajectory for the closed-loop system (8)-(13).

ii) if $\mathbf{A} \mathbf{1}$ holds then, for any $\rho>0$, there exists $\vartheta>0$ such that, for any $M$ with $|\dot{M}|_{s}<\vartheta,(p, \dot{p})=\left(p_{r}, \dot{p}_{r}\right)$ is a (uniformly) $A S$ and LES equilibrium trajectory for the closed-loop system (8)-(13) with $C D$ containing $\mathcal{A}_{\rho}:=\{(p, \dot{p})(0):|(\tilde{p}(0), \tilde{v}(0))| \leq$ $\rho\}$, with $|(\tilde{p}, \tilde{v})|:=\sqrt{|\tilde{p}|^{2}+|\tilde{v}|^{2}}$.

Proposition 1 provides bounded feedback laws that ensure global (or semi-global) stability in the presence of uncertain measurements for System (8). Condition (12) can be used to specify admissible control gains given upper bounds on the uncertainties (the norm of $M$ and of its skew-symmetric part $M_{a}$ ). This kind of result is very similar to classical Robust Stability theory for linear systems (Doyle et al., 1992, Ch. 4), Doyle and Stein (1981), Chen and Desoer (1982), where the objective is to guarantee stability for a set of plants that satisfy some uncertainty bound w.r.t. a nominal system. In our case, uncertainty corresponds to the difference between $M$ and the identity matrix. If the uncertainty is small (which implies in particular that $M_{a}$ is close to the zero matrix and $|M|_{i} \approx 1$ ), then Condition (12) puts little constraints on the control gains. If $M_{a}$ is large, however, large values of $k_{2}$ are needed. In summary, Proposition 1 can provide stability guarantees given an a priori bound on uncertainties. Note also that when $M$ is constant, global asymptotic stability can be obtained while only semi-global asymptotic stability is obtained when $M$ varies with time (Case $i i)$ ).

\section{Underactuated case with velocity measurements}

Let us consider the control system (5), and assume that the following measurements are available:

$$
\tilde{\sigma}, \gamma:=g R^{T} e_{3}, v, \omega
$$

Compared to the fully actuated case, there are two additional measurements, i.e. $\gamma$ and $\omega$. The latter is typically obtained from the gyrometers of an IMU, while the former is obtained by fusing accelerometer and gyrometer measurements (see, e.g. Mahony et al. (2012)). Let $q:=\operatorname{Re}_{3}$ denote the thrust direction, so that the first equality in (5) can also be written as

$$
\ddot{p}=-u q+g e_{3}=R\left(-u e_{3}+\gamma\right)
$$

If $\mathbf{A 3}$ is satisfied then, along the reference position trajectory $p_{r}$ the thrust direction is well defined (this is no longer true if $\ddot{p}_{r}=$ $g e_{3}$ since any thrust direction $q$ is solution to (15) for $u=0$ ). More precisely, assuming that $u$ is positive, this reference thrust direction is

$$
q_{r}:=\frac{g e_{3}-\ddot{p}_{r}}{\left|g e_{3}-\ddot{p}_{r}\right|}
$$

Proposition 2 Let $\mathrm{sat}_{\delta}, \overline{\mathrm{sat}}_{\bar{\delta}}$ denote two saturation functions. Consider control gains $k_{1}, k_{2}>0$ satisfying (12) with $C_{\delta}$ defined by (2), and the additional condition

$$
k_{1} \delta_{M}+k_{2} \bar{\delta}_{M}+\left|\ddot{p}_{r}\right|_{s}<g
$$


Define a dynamic augmentation

$$
\dot{\eta}=\eta \times \omega-k_{3}(\eta-\tilde{\sigma}), \quad k_{3}>0
$$

together with the control law

$$
\left\{\begin{array}{l}
\omega_{1}=-\frac{k_{4}|\mu| \mu_{2}}{\left(|\mu|+\mu_{3}\right)^{2}}-\frac{1}{|\mu|^{2}} \mu^{T} S\left(e_{1}\right) \varpi \\
\omega_{2}=\frac{k_{4}|\mu| \mu_{1}}{\left(|\mu|+\mu_{3}\right)^{2}}-\frac{1}{|\mu|^{2}} \mu^{T} S\left(e_{2}\right) \varpi \\
u=\mu_{3}
\end{array}\right.
$$

with $k_{4}>0$ and $\mu$ and $\varpi$ defined by:

$$
\begin{aligned}
\mu:= & \gamma+k_{1} \operatorname{sat}_{\delta}(\eta)+k_{2} \overline{\operatorname{sat}}_{\bar{\delta}}(\tilde{v})-a_{r} \\
\varpi:= & -k_{1} k_{3}\left[\mathrm{~s}_{\delta}\left(|\eta|^{2}\right) I_{3}+2 \mathrm{~s}_{\delta}^{\prime}\left(|\eta|^{2}\right) \eta \eta^{T}\right](\eta-\tilde{\sigma}) \\
& +k_{2}\left[\overline{\mathrm{s}}_{\tilde{\delta}}\left(|\tilde{v}|^{2}\right) I_{3}+2 \overline{\mathrm{s}}_{\bar{\delta}}^{\prime}\left(|\tilde{v}|^{2}\right) \tilde{v} \tilde{v}^{T}\right]\left(\gamma-u e_{3}\right)
\end{aligned}
$$

with $\tilde{v}$ and $a_{r}$ defined by (10) and (11). Then,

i) if A1, A2, and A3 hold then, there exists $\underline{k}_{3}>0$ such that, for any $k_{3}>\underline{k}_{3},(p, \dot{p}, q, \eta)=\left(p_{r}, \dot{p}_{r}, q_{r}, 0\right)$ is a (uniformly) $A S$ and LES equilibrium trajectory for the closed-loop system (5)-(18)-(19)-(20) with $C D$

$$
\mathcal{A}:=\left\{(p, \dot{p}, q, \eta)(0): \mu(0) \neq-|\mu(0)| e_{3}\right\}
$$

ii) if $\mathbf{A} 1$ and $\mathbf{A} 3$ hold then, for any $\rho>0$, there exist $\vartheta, \underline{k}_{3}>$ 0 such that, for any $k_{3}>\underline{k}_{3}$ and any $M$ with $|\dot{M}|_{s}<\vartheta,(p, \dot{p}, \bar{q}, \eta)=$ $\left(p_{r}, \dot{p}_{r}, q_{r}, 0\right)$ is an AS and LES equilibrium trajectory for the closed-loop system (5)-(18)-(19)-(20) with CD containing

$$
\begin{aligned}
\mathcal{A}_{\rho}:=\{(p, \dot{p}, q, \eta)(0):|(\tilde{p}(0), \tilde{v}(0), \eta(0))| & \leq \rho, \\
\mu(0) & \left.\neq-|\mu(0)| e_{3}\right\}
\end{aligned}
$$

Let us discuss the links between this result and Proposition 1. First, except for the $\gamma$ term, $\mu$ in (20) is reminiscent of the control law (13) with $\tilde{\sigma}$ replaced by $\eta$. In view of (18), this latter variable can be viewed as a "filtered value" of $\tilde{\sigma}$. The important point is that $\dot{\eta}$ is known, since it is explicitly given by (18), while the time derivative of $\tilde{\sigma}$ is not, since $M$ is unknown. Then, the control inputs $u, \omega_{1}, \omega_{2}$ are defined so that $\mu$ converges to $|\mu| e_{3}$. This implies, using the second equality in (15), that $\ddot{p}$ converges to $R\left(-k_{1} \operatorname{sat}_{\delta}(\eta)-k_{2} \overline{\operatorname{sat}}_{\bar{\delta}}(\tilde{v})+a_{r}\right)$. This expression is the same as (8)-(13) with $\tilde{\sigma}$ being replaced by $\eta$. This explains the relation between Propositions 1 and 2. Finally,

$$
\begin{aligned}
& \left|k_{1} \operatorname{sat}_{\delta}(\eta)+k_{2} \overline{\operatorname{sat}}_{\delta}(\tilde{v})-a_{r}\right| \\
\leq & k_{1} \delta_{M}+k_{2} \bar{\delta}_{M}+\left|\ddot{p}_{r}\right|_{s}<g=|\gamma|
\end{aligned}
$$

where the second inequality comes from (17). This inequality implies that:

Lemma $1 \mu(0) \neq-|\mu(0)| e_{3}$ if

$$
\gamma_{3}(0)>-\sqrt{g^{2}-\left(k_{1} \delta_{M}+k_{2} \bar{\delta}_{M}+\left|\ddot{p}_{r}\right|_{s}\right)^{2}}
$$

Since $\gamma_{3}=g q_{3}$, Lemma 1 implies that in both cases $i$ ) and $i i$ ) the $C D$ in roll/pitch contains the upper hemisphere. Note that Condition (24) is conservative. Thus, in both cases $i$ ) and ii), a large stability domain in orientation is guaranteed. Global stability is ruled out because $q$ belongs to a compact set (i.e., the unit sphere). If (24) is satisfied, there is no constraint on the initial values of position, linear velocity, and dynamic augmentation variables in case $i$ ) (see (21)). In case $i i$ ), initial values can be made arbitrarily large under conditions on $k_{3}$ and $\dot{M}$.

\subsection{Simplified control law}

Another control expression with similar robustness properties is proposed next. It involves a simpler control expression and does not require the dynamic extension (18).

Proposition 3 With the notation of Prop. 2, assume that the following extra condition on the gains $k_{1}, k_{2}$ is satisfied:

$$
k_{1} \delta_{M}+k_{2} \bar{\delta}_{M}+\left|\ddot{p}_{r}\right|_{s} \leq g(1-\varkappa), \quad 0<\varkappa<1
$$

and define the control law as:

$$
\left\{\begin{array}{l}
\omega_{1}=-k_{4} \mu_{2}, \quad \omega_{2}=k_{4} \mu_{1} \\
u=\mu_{3}
\end{array}\right.
$$

with

$$
\mu:=\gamma+k_{1} \operatorname{sat}_{\delta}(\tilde{\sigma})+k_{2} \overline{\operatorname{sat}}_{\bar{\delta}}(\tilde{v})-a_{r}
$$

Then,

i) If $\mathbf{A 1}, \mathbf{A 2}$, and $\mathbf{A} 3$ hold then, for any $\rho>0$, there exists $k_{4, \rho}>0$ such that, for any $k_{4}>k_{4, \rho},(p, \dot{p}, q)=\left(p_{r}, \dot{p}_{r}, q_{r}\right)$ is a (uniformly) AS and LES equilibrium trajectory for the closedloop system (5)-(26)-(27), with CD containing

$$
\begin{aligned}
\mathcal{A}_{\rho}:=\{(p, \dot{p}, q)(0):|\tilde{v}(0)|<\rho, \\
\left.\left|\left(\mu_{1}(0), \mu_{2}(0)\right)\right|<g \varkappa / \sqrt{2}, \mu_{3}(0)>0\right\}
\end{aligned}
$$

ii) If $\mathbf{A 1}$ and $\mathbf{A} \mathbf{3}$ hold then, for any $\rho>0$, there exist $\vartheta, \underline{k}_{4}>0$ such that, for any $k_{4}>\underline{k}_{4}$ and any $M$ with $|\dot{M}|_{s}<\vartheta$, $(p, \dot{p}, q)=\left(p_{r}, \dot{p}_{r}, q_{r}\right)$ is an AS and LES equilibrium trajectory for the closed-loop system (5)-(26)-(27) with CD containing

$$
\begin{aligned}
\mathcal{A}_{\rho}:=\left\{(p, \dot{p}, q)(0):|\tilde{p}(0)|<\rho,|\tilde{v}(0)|<\bar{\delta}_{m} / 2,\right. \\
\left.\left|\left(\mu_{1}(0), \mu_{2}(0)\right)\right|<g \varkappa / \sqrt{2}, \mu_{3}(0)>0\right\}
\end{aligned}
$$

The main assets of Proposition 3 are a large stability domain, robustness to position measurement uncertainties, and the simplicity of the control expression. Concerning the latter aspect, the fact that the control expression is essentially linear (modulo saturation functions) is clearly an asset with respect to the control law of Proposition 2, e.g., when considering effects of measurement noise. Another asset is related to the extension of the present analysis to the full model (4) (i.e., considering $\Gamma$ as control input instead of $\omega$ ). A classical solution in this case would be a linear torque feedback with feedforward action. Computing the feedforward action requires to differentiate angular velocity inputs proposed above. Differentiating $\omega_{1}, \omega_{2}$ in (26) is much simpler than for (19) and requires much less information. In addition, one may want in this case to replace $\tilde{\sigma}$ in (27) by $\eta$ given by (18) since $\dot{\eta}$ is known. Additional work is needed for the stability analysis of such a torque control law and this issue is left for future research.

Another common approach to extend the controller from kinematics to dynamics is to use a high gain controller: $\Gamma=$ $-J \omega \times \omega-k J\left(\omega-\omega^{d}\right)$ with $k$ chosen large enough and $\omega^{d}$ the kinematic controller Hua et al. (2009); Brescianini and D'Andrea (2016). This simple solution is motivated by a time separation argument. To the authors' knowledge its stability analysis remains an open issue. 


\section{Extensions to GPS-denied environments}

This section considers extension of the results of Section 4 to velocity-free scenarios, i.e., when velocity $v$ is not measured. This is a challenging problem and we only consider a special case of the general framework addressed in Section 4. We will show in the application section, however, that this special case covers important scenarios.

Let $v_{M}:=R^{T} M \dot{p}$. Assume that $M$ and $p_{r}$ are constant values. Then, $v_{M}=R^{T} M \dot{\tilde{p}}$ and it follows from (6) that

$$
\begin{cases}\dot{\tilde{\sigma}} & =\tilde{\sigma} \times \omega+v_{M} \\ \dot{v}_{M} & =v_{M} \times \omega+R^{T} M \ddot{p}\end{cases}
$$

We want to obtain an estimation of the non-measured variable $v_{M}$. To this purpose, consider the following observer:

$$
\left\{\begin{array}{l}
\dot{\hat{\sigma}}=\hat{\sigma} \times \omega+\hat{v}_{M}-2 \alpha k(\hat{\sigma}-\tilde{\sigma}) \\
\dot{\hat{v}}_{M}=\hat{v}_{M} \times \omega-k^{2}(\hat{\sigma}-\tilde{\sigma})
\end{array}\right.
$$

Proposition 4 Let $e_{\sigma}:=\hat{\sigma}-\tilde{\sigma}$ and $e_{v}:=\hat{v}_{M}-v_{M}$ denote the estimation errors. Assume that there exists a constant $C$ such that, for any initial condition, $|\ddot{p}(t)| \leq C, \forall t$. Then, for any $\varepsilon>0$ and any $\alpha>0$ there exists $k_{m}>0$ such that, for any $k \geq k_{m}$ and any initial condition $\left(\hat{\sigma}(0), \hat{v}_{M}(0)\right),\left|e_{\sigma}\right|+\left|e_{v}\right|$ is ultimately bounded by $\varepsilon$.

From Proposition 4 a good estimate of $v_{M}$ can be built from the measurement $\tilde{\sigma}$ if $\ddot{p}$ is bounded. Note that the control laws derived in Section 4 ensure the boundedness of $\ddot{p}$. This suggests to use such control laws with $\hat{v}_{M}$ as velocity input in place of $v$. In the rest of this section, we focus on the stability analysis of these control laws with $v_{M}$ as velocity input. By doing so, we neglect the discrepancy between $\hat{v}_{M}$ and $v_{M}$ knowing that, from Proposition 4, it can be made arbitrarily small ultimately. Stability analysis of the couple controler/observer is left for future studies.

The rest of this section will invoke Assumptions 1, 2, and 4. Note that, when $p_{r}$ is a constant vector, the control law $u$ in (13) can be written as $u:=k_{1} \operatorname{sat}_{\delta}(\tilde{\sigma})+k_{2} \overline{\operatorname{sat}}_{\bar{\delta}}(v)$. The following propositions show that the results of Section 4 can be extended to the case of velocity measurements $v_{M}$ with minor modifications. Due to space limitations, we only address extension of Propositions 1 and 3.

Proposition 5 Let $\mathrm{sat}_{\delta}, \overline{\mathrm{sat}}_{\bar{\delta}}$ denote two $\mathbb{R}^{3}$-valued saturation functions with associated functions $\mathrm{s}_{\delta}, \overline{\mathrm{s}}_{\bar{\delta}}$. Consider control gains $k_{1}, k_{2}>0$ such that

$$
\left\{\begin{array}{l}
k_{2}^{2}|M|_{i}^{2}>k_{1}\left|M_{a} M^{-1}\right|_{s} \max \left\{C_{\delta}|M|_{s},\left|M_{a} M^{-1}\right|_{s}|M|_{i}\right\} \\
k_{2} \bar{\delta}_{m}|M|_{i}>k_{1} \delta_{M}|M|_{s}
\end{array}\right.
$$

and define the control law

$$
u:=k_{1} \operatorname{sat}_{\delta}(\tilde{\sigma})+k_{2} \overline{\operatorname{sat}}_{\bar{\delta}}\left(v_{M}\right)
$$

If A1, A2, and $\mathbf{A 4}$ hold then $(p, \dot{p})=\left(p_{r}, 0\right)$ is a (uniformly) GAS and LES equilibrium point for the closed-loop system (8)(33).
Proposition 6 Let $\mathrm{sat}_{\delta}, \overline{\mathrm{sat}}_{\bar{\delta}}$ denote two saturation functions. Consider control gains $k_{1}, k_{2}>0$ satisfying (32) with $C_{\delta}$ be defined by (2), and the additional condition

$$
k_{1} \delta_{M}+k_{2} \bar{\delta}_{M} \leq g(1-\varkappa), \quad 0<\varkappa<1
$$

and consider the control law (26) with

$$
\mu:=\gamma+k_{1} \operatorname{sat}_{\delta}(\tilde{\sigma})+k_{2} \overline{\operatorname{sat}}_{\bar{\delta}}\left(v_{M}\right)
$$

If A1, A2, and A4 hold then, for any $\rho>0$, there exists $k_{4, \rho}>0$ such that, for any $k_{4}>k_{4, \rho},(p, \dot{p}, q)=\left(p_{r}, 0, q_{r}\right)$ is a (uniformly) AS and LES equilibrium point for the closed-loop system (5)-(26)-(35), with CD containing

$$
\begin{aligned}
\mathcal{A}_{\rho}:=\left\{(p, \dot{p}, q)(0):\left|v_{M}(0)\right|<\rho,\right. \\
\left.\left|\left(\mu_{1}(0), \mu_{2}(0)\right)\right|<g \varkappa / \sqrt{2}, \mu_{3}(0)>0\right\}
\end{aligned}
$$

\section{Application to visual servoing of UAVs}

Micro UAVs (MAVs) are increasingly used for surveillance and inspection applications. Controlling such systems through vision sensors is an important issue. A first solution consists in using a stereo vision system. When MAVs operate far from ground/obstacles, however, the advantage of a stereo system w.r.t. a mono-camera system is questionable since the quality of depth-estimation is then poor due to the short baseline. In fact, a mono-camera system can be preferred for simplicity and because image processing can be performed at a higher framerate. Thus, much attention has been paid to mono-camera visual servoing of UAVs (see, e.g., Saripalli et al. (2003); Conte and Doherty (2008); Caballero et al. (2009); Cunha et al. (2011); Mondragon et al. (2010)). These approaches often rely on the estimation of the so-called "Homography matrix", which embeds information on the camera's pose. However, the relation between the Homography matrix and the pose involves quantities that may be poorly known. This issue is often resolved in the litterature by considering restrictive scenarios (e.g., perfectly calibrated camera, camera observing an horizontal ground knowledge of the vehicle's altitude, etc). We show next that our results provide stability guarantees for a much larger range of scenarios.

\subsection{Preliminary recalls and problem statement}

Consider an underactuated UAV with a video camera facing a planar target. Suppose that a "reference" picture of this target taken at a reference pose is known. This reference pose is represented on Fig. 1 by the reference frame $\mathcal{R}^{*}$. We assume that this pose is a possible equilibrium for the dynamics of this underactuated vehicle, meaning that the $z$ axis of $\mathcal{R}^{*}$ is vertical. Otherwise, asymptotic stablization of this pose is not possible. We also assume that the optical center of the camera corresponds to the vehicle's center of mass and the optical axis corresponds to the $x$-axis of $\mathcal{R}^{*}$. At every time, the "current" picture of the target, taken at the current pose represented by the frame $\mathcal{R}$, is compared to the reference one. From there, the homography matrix is computed (see e.g. Ma et al. (2003)). 
This matrix, which transforms the target's points coordinates from the reference pose to the current pose, is

$$
H:=R^{T}-\frac{1}{d^{*}} R^{T} p n^{* T}
$$

with $d^{*}$ the distance from the UAV reference position to the target plane and $n^{*}$ the normal to this plane expressed in the reference frame. Both $d^{*}$ and $n^{*}$ are unknown and thus unavailable for the control design. We show next that for any orientation of the visual target one can extract from $H$ position measurements of the form (6). The case of a non-vertical target is first briefly described. Then, the case of a vertical target is studied in more details.

\subsection{Non-vertical target}

In this case, $n_{3}^{*}:=n^{* T} e_{3}>0$ and it follows from (37) that $\mathrm{He}_{3}=R^{T} e_{3}-\left(n_{3}^{*} / d^{*}\right) R^{T} p=\frac{\gamma}{g}-\frac{n_{3}^{*}}{d^{*}} R^{T} p$. As recalled in Section $4, \gamma$ is usually estimated from the UAV's IMU. By substracting $\frac{\gamma}{g}$ to $\mathrm{He}_{3}$, one obtains the measurement $\tilde{\sigma}=\frac{n_{3}^{*}}{d^{*}} R^{T} p=R^{T} M p$ with $M=\frac{n_{3}^{*}}{d^{*}} I>0$. Since $M$ is constant and diagonal, it satisfies all the conditions in Assumptions 1,2, and 4. All the results of Sections 4 and 5 apply and yield stability conditions in term of the control gains and the constant number $n_{3}^{*} / d^{*}$.

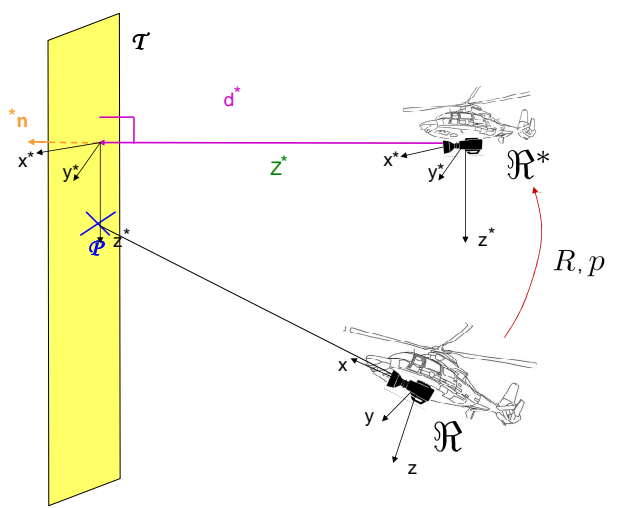

Figure 1: Problem scheme

\subsection{Vertical target, i.e. $n_{3}^{*}=0$}

This case is of interest in many inspection applications since many man-made buildings are vertical. Let

$$
\tilde{\sigma}:=\left(H e_{2}\right) \times\left(H e_{3}\right)-H e_{1}, \quad \gamma:=g H e_{3}
$$

From (37) and the assumption $n_{3}^{*}=0$, one can verify that

$$
\tilde{\sigma}=R^{T} M\left(\frac{n^{*}}{d^{*}}\right) p, \quad \gamma=g R^{T} e_{3}
$$

with $M(\tau):=\tau_{1} I+S\left(\tau_{2} e_{3}\right)$. Thus, $\tilde{\sigma}$ and $\gamma$ satisfy Eq. (6), (14) with $p_{r}=0$. Note that $M_{s}(\tau)=\tau_{1} I$ and $M_{a}(\tau)=S\left(\tau_{2} e_{3}\right)$ commute for any $\tau$. Thus, like for a non-vertical target, $M$ satisfies all the conditions in Assumptions 1, 2, and 4. We detail below application of our results to fixed-point stabilization and way-points navigation.
Vision based point stabilization. Let us first address the stabilization of the UAV at the reference pose. From (39), Proposition 2-i) applies directly with $M=M\left(\frac{n^{*}}{d^{*}}\right)$ provided the gain conditions (12) and (17) are satisfied. We deduce that the control law (18)-(19) ensures asymptotic stabilization of the reference pose (with global convergence domain in position/velocity) if:

$$
\begin{array}{ll}
\text { a) } n_{1}^{*}, k_{1}, k_{2}>0 & \text { b) } k_{2} \bar{\delta}_{m}>k_{1} \delta_{M} \\
\text { c) } k_{1} \delta_{M}+k_{2} \bar{\delta}_{M}<g & \text { d) } n_{1}^{*} d^{*} k_{2}^{2}>k_{1}\left|n_{2}^{*}\right|\left(\left|n_{2}^{*}\right|+\frac{2 n_{1}^{*}}{3 \sqrt{3}}\right)
\end{array}
$$

Condition $n_{1}^{*}>0$, which ensures that $M>0$, means that the camera is "facing" the target at the reference pose (obvious condition in practice). Given bounds on the uncertain parameters $d^{*}, n^{*}$, i.e., $d^{*} \in\left[\underline{d}^{*}, \bar{d}^{*}\right], n_{1}^{*} \in\left[\underline{n}_{1}^{*}, 1\right]$, Condition d) can be replaced by: $\underline{n}_{1}^{*} \underline{d}^{*} k_{2}^{2}>k_{1}(1+2 /(3 \sqrt{3}))$. Thus, one obtains stability conditions on the control parameters given bounds on the uncertain parameters $d^{*}, n^{*}$.

Yaw control. The yaw degree of freedom is not involved in the stabilization objective. In practice, it matters to keep the target inside the field of view of the camera. We propose the following yaw control law: $\omega_{3}=k_{5} H_{21}$. Upon convergence of the position, velocity, roll and pitch errors to zero, the yaw dynamics will be close to $\dot{\psi} \approx-k_{5} \sin \psi$, thus ensuring the convergence of $\psi$ to zero unless $\psi(0)=\pi$ (a case contradictory with the visibility assumption).

Visual-based way-points navigation. Consider a sequence of reference images of a planar scene taken from different reference frames (hereafter referred to as way-points). The objective is to make the UAV navigate along this sequence of way-points. Without loss of generality, we consider two way-points. From the two reference images and the current image, one can define two homography matrices, from which are computed two uncertain relative position measurements $\tilde{\sigma}_{1}, \tilde{\sigma}_{2}$ as defined by Eq. (38). Let $p_{i}$ denote the position vector of the current frame w.r.t. the $i$-th reference frame and $R_{i}$ denote the rotation matrix from the current frame to the $i$-th reference frame. If $\chi$ (resp. $\chi_{i}^{*}$ ) denotes the coordinate vector of a point of the scene in the current frame (resp. in the $i$-th reference frame), then $\chi_{1}^{*}=R_{1} \chi+p_{1}$ and $\chi_{2}^{*}=R_{2} \chi+p_{2}$. The transformation between the two reference frames is defined as $\chi_{2}^{*}=\bar{R} \chi_{1}^{*}+\bar{p}$ where $\bar{R}$ is a constant matrix and $\bar{p}$ is a constant vector, and one has $R_{2}=\bar{R} R_{1}$ and $p_{2}=\bar{R} p_{1}+\bar{p}$. We implicitly define a reference trajectory by considering a time-varying interpolation $\tilde{\sigma}$ of $\tilde{\sigma}_{1}$ and $\tilde{\sigma}_{2}: \tilde{\sigma}:=(1-\lambda(t)) \tilde{\sigma}_{1}+\lambda(t) \tilde{\sigma}_{2}$ with $\lambda$ an increasing function ranging over $[0,1]$. Consider the generic case where both parameters $n^{*}, d^{*}$ change between the two reference images. Then, we deduce from the above relations that

$$
\begin{cases}\tilde{\sigma} & =R_{1}^{T} \bar{M}\left(p_{1}-p_{r}\right) \\ \bar{M}(t) & :=(1-\lambda(t)) M\left(\frac{n_{1}}{d_{1}}\right)+\lambda(t) \bar{R}^{T} M\left(\frac{n_{2}}{d_{2}}\right) \bar{R} \\ p_{r}(t) & :=-\lambda(t) \bar{M}(t)^{-1} \bar{R}^{T} M\left(\frac{n_{2}}{d_{2}}\right) \bar{p}\end{cases}
$$

Thus, $\tilde{\sigma}$ is of the form of Eq. (6) with $M(t)$ replaced by $\bar{M}(t)$. Proposition 2-ii) can then be used to ensure semi-global stability of this non-stationary reference trajectory. Note that the 
existence of $|\bar{M}|_{i}>0,|\bar{M}|_{s}<+\infty$ and $|\dot{\bar{M}}|_{s}<+\infty$ in Assumption A1 for the matrix $\bar{M}$ (.) follows from the fact that $M>0$ and $\lambda(t) \in[0,1]$. Thus, the linear interpolation of $\tilde{\sigma}_{1}$ and $\tilde{\sigma}_{2}$ implicitly defines a reference trajectory with compact image set. Compacity plays a key role in ensuring that Assumption A1 is satisfied.

\section{Simulation results}

We illustrate the results of this paper for the visual servoing applications of Section 6, in the case of a vertical visual target. We first consider the fixed-point stabilization problem. The initial conditions and scene parameters at the reference pose are:

$$
\left\{\begin{array}{l}
p_{0}=(-5.5 \mathrm{~m} ; 1.2 \mathrm{~m} ; 1.3 \mathrm{~m})^{T} \\
v_{0}=\left(-2.3 \mathrm{~m} \cdot \mathrm{s}^{-1} ;-1.6 \mathrm{~m} \cdot \mathrm{s}^{-1} ; 0.4 \mathrm{~m} \cdot \mathrm{s}^{-1}\right)^{T} \\
\phi_{0}=-0.5^{\circ}, \theta_{0}=11.4^{\circ}, \psi_{0}=4.1^{\circ} \\
n^{*}=(0.7 ;-0.71 ; 0)^{T}, d^{*}=3 \mathrm{~m}
\end{array}\right.
$$

For the simulation reported on Fig. 2, the control law of Prop. 2 is used with $\eta(0)=0$. For the simulation reported on Fig. 3, the simplified controller of Prop. 3 is used. For the simulation reported on Fig. 4, the velocity-free controller of Prop. 6 is used with $v_{M}$ replaced by $\hat{v}_{M}$ and $\hat{v}_{M}$ the output of the observer (31). The control gains are $\left[k_{1}, k_{2}, k_{3}, k_{4}, k_{5}\right]=[1,1.5,1,1,1]$ and the saturation functions $\mathrm{sat}_{\delta}, \overline{\mathrm{sat}}_{\bar{\delta}}$ are defined from the expression (3) of the associated functions $\mathrm{s}_{\delta}, \bar{s}_{\bar{\delta}}$, with $\delta=[.9 ; 1], \bar{\delta}=$ $[1 ; 1.1]$. The gains of the observer (31) are $k=7, \alpha=0.7$ and $\hat{\sigma}(0)=\hat{v}_{M}(0)=0$. All controllers stabilize the system. Transient behaviors are qualitatively similar but differences can be noticed. With respect to the control law of Prop. 2, the simplified control law of Prop. 3 yields a shorter settling time, less overshoot, and smaller angular velocities. The velocityfree control law of Prop. 6 yields results similar to that of the first simulation but the convergence of the angular velocity to zero is slower. From Fig. 4 one can also conjecture asymptotic stability of the couple observer/controller, i.e., asymptotic convergence of $\hat{v}_{M}$ to $v_{M}$ and of the tracking error to zero.
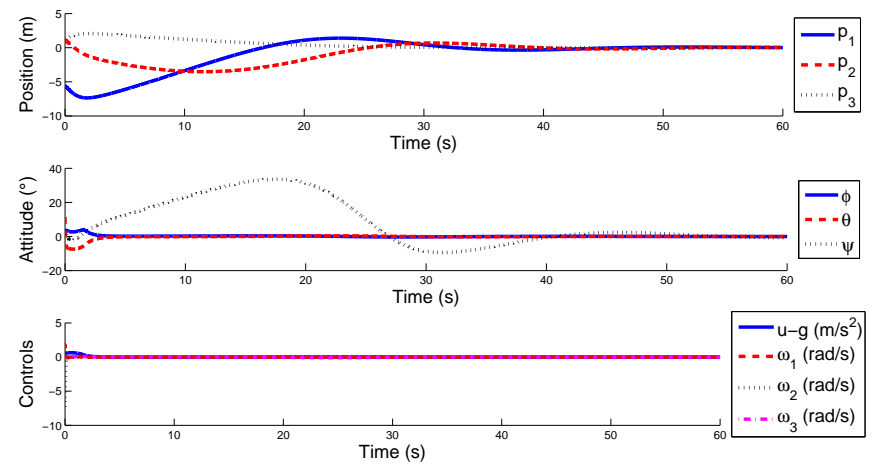

Figure 2: Fixed-point stabilization: control law of Proposition 2.

Validation of the visual way-points navigation, with the control law of Prop. 3 and $\tilde{\sigma}$ defined by (40), is presented on
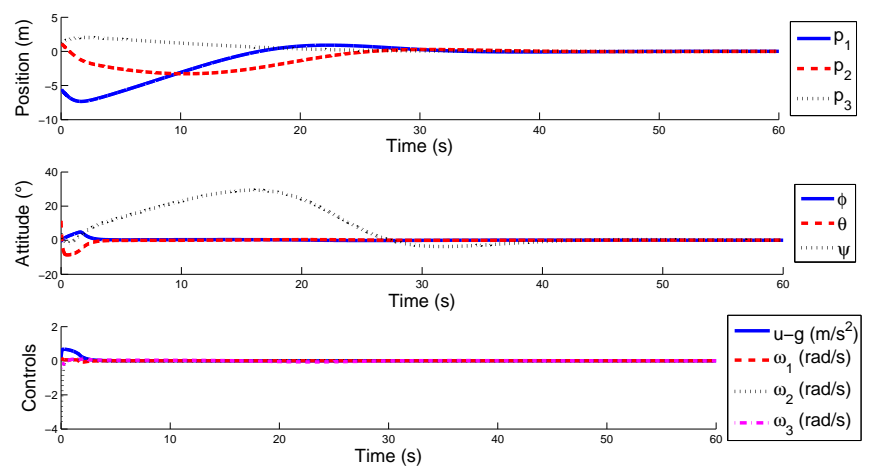

Figure 3: Fixed-point stabilization: control law of Proposition 3.
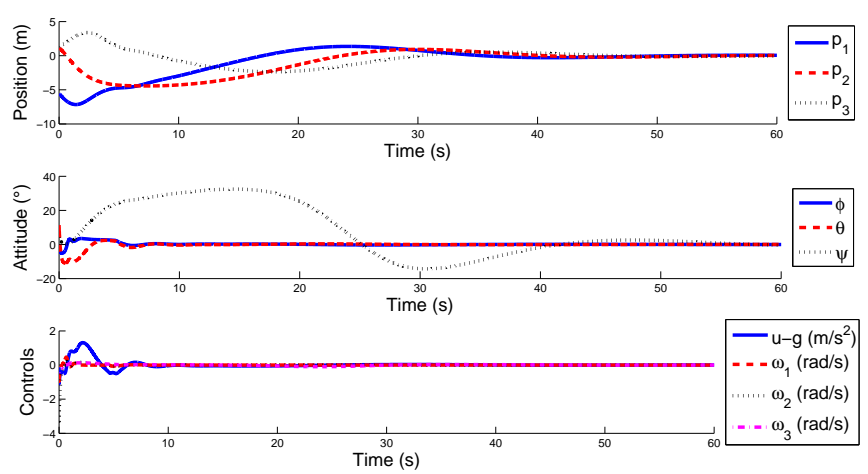

Figure 4: Fixed-point stabilization: control law of Proposition 6.

Fig. 5. The interpolation function $\lambda$ is defined as: $\lambda(t)=0$ for $t \leq t_{s}, \lambda(t)=1$ for $t \geq t_{e}$ and $\lambda(t)=\frac{t-t_{s}}{t_{e}-t_{s}}$ for $t_{s}<t<t_{e}$ with $t_{s}=50 \mathrm{~s}, t_{e}=80 \mathrm{~s}$. Initial conditions and scene parameters for the first image are still given by (41). Scene parameters for the second image (i.e., second way-point) are $n^{*}=$ $(0.87,-0.5,0)^{T}, d^{*}=1 / 3 \mathrm{~m}$. The position vector between the two way-points is defined by $\bar{p}=(-5,10,20)^{T}$. Let us notice the smooth transition and small control input values on the transition interval.
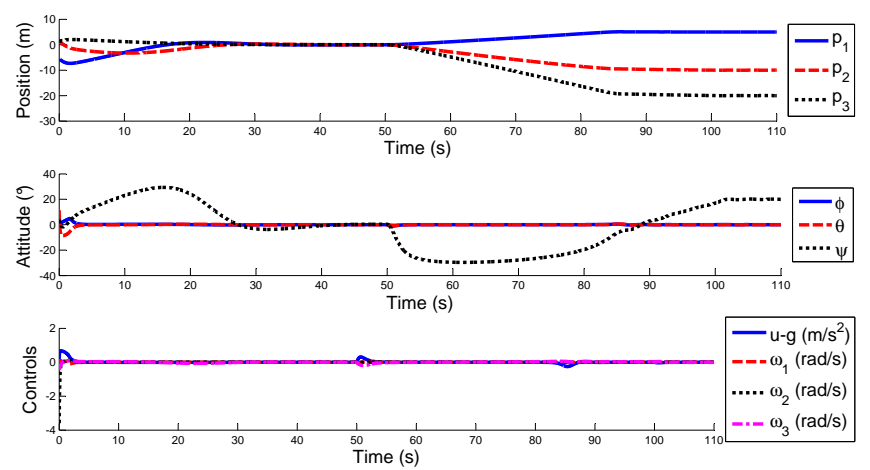

Figure 5: Way-points navigation: control law of Proposition 3. 


\section{Conclusion}

We have proposed a feedback control design and robustness analysis for the stabilization of a class of underactuated vehicles with uncertain position measurements. Strong stability results have been obtained for a large class of position measurements uncertainties and sufficient stability conditions on the control gains have been derived in relation with the norm of the uncertainties. We have shown direct applications of these results to UAVs for two visual servoing problems: fixed-point stabilization and visual way-points navigation w.r.t. a planar scene. Simulation results support the proposed analysis.

\section{Proof of Propositions}

Proof of Proposition 1: From (6), $x:=R \tilde{\sigma}=M \tilde{p}$. From (9)-(11) $y:=R \tilde{v}=\dot{\tilde{p}}$. Thus, $\dot{x}=M y+\dot{M} \tilde{p}$ and, from (8), (11) and (13), $\dot{y}=-R\left(k_{1} \operatorname{sat}_{\delta}(\tilde{\sigma})+k_{2} \overline{\operatorname{sat}}_{\bar{\delta}}(\tilde{v})\right)$. From Definition 1-i), $R \operatorname{sat}_{\delta}(\xi)=\operatorname{sat}_{\delta}(R \xi)$ for any $\xi$. Therefore, the closed-loop system in $(x, y)$ coordinates is given by:

$$
\left\{\begin{aligned}
\dot{x} & =M y+\dot{M} \tilde{p} \\
\dot{y} & =-k_{1} \operatorname{sat}_{\delta}(x)-k_{2} \overline{\operatorname{sat}}_{\bar{\delta}}(y)
\end{aligned}\right.
$$

Lemma 2 Let $y$ denote a solution of the equation

$$
\dot{y}=-k_{1} \operatorname{sat}_{\delta}(x)-k_{2} \overline{\operatorname{sat}}_{\bar{\delta}}(y)+\varrho(t)
$$

with $x$ any time-function and $\varrho$ a bounded and continuous timefunction such that, for some $t_{1} \geq 0$ and $c_{\varrho}>0$,

$$
\varrho(t) \mid \leq k_{2} \bar{\delta}_{m}-k_{1} \delta_{M}-c_{\varrho}, \quad \forall t \geq t_{1}
$$

Then, there exists a continuous function $T$ such that, for $t \geq$ $T(y(0)), \overline{\operatorname{sat}}_{\bar{\delta}}(y(t))=y(t)$, i.e., the function $\overline{\mathrm{sat}}_{\bar{\delta}}$ desaturates after time $T(y(0))$.

Property i): Assumption A2 implies that $\dot{M}=0$. Applying Lemma 2 to the second equation in (42) with $\varrho \equiv 0$ and using the second condition in (12), one deduces that along any solution of System (42) the function $\overline{\mathrm{sat}}_{\bar{\delta}}$ desaturates after some time and the solution then satisfies:

$$
\left\{\begin{array}{l}
\dot{x}=M y \\
\dot{y}=-k_{1} \operatorname{sat}_{\delta}(x)-k_{2} y=-k_{1} \mathrm{~s}_{\delta}\left(|x|^{2}\right) x-k_{2} y
\end{array}\right.
$$

Consider the CLF (Candidate Lyapunov Function) $V$ defined by:

$$
\begin{aligned}
V(x, y)= & k_{1} \int_{0}^{|x|^{2}} \mathrm{~s}_{\delta}(\tau) d \tau+y^{T} M y \\
& +\frac{2 k_{1}}{k_{2}} \mathrm{~s}_{\delta}\left(|x|^{2}\right) x^{T} M_{a} y+\kappa \mathrm{s}_{\delta}\left(|x|^{2}\right) x^{T} y \\
= & k_{1} \int_{0}^{|x|^{2}} \mathrm{~s}_{\delta}(\tau) d \tau+y^{T} M y \\
& +\frac{2 k_{1}}{k_{2}} \operatorname{sat}_{\delta}(x)^{T} M_{a} y+\kappa \operatorname{sat}_{\delta}(x)^{T} y
\end{aligned}
$$

where $\kappa$ is a constant positive number. We show that $V$ is a Lyapunov function for $\kappa$ small enough. Let

$$
\left\{\begin{aligned}
\bar{\kappa} & :=k_{2}^{2}|M|_{i}-k_{1}\left|M_{a}\right|_{s}|M|_{s} C_{\delta}>0 \\
\kappa_{1} & :=2 \sqrt{k_{1}|M|_{i}}-2 \frac{k_{1}}{k_{2}}\left|M_{a}\right|_{s}>0 \\
\kappa_{2} & :=\frac{2 \bar{\kappa}}{k_{2}|M|_{s} C_{\delta}}>0 \\
\kappa_{3} & :=\frac{k_{2}}{k_{2}|M|_{s} C_{\delta}+\frac{k_{2}^{3}}{4 k_{1}}}>0 \\
0<\kappa & <\min \left\{\kappa_{1}, \kappa_{2}, \kappa_{3}\right\}
\end{aligned}\right.
$$

where positivity of $\bar{\kappa}, \kappa_{1}$ follows from (12) and positivity of $\kappa_{2}, \kappa_{3}$ is a consequence thereof. We first prove that $V$ is positive definite and proper. Integrating by part and using the fact that $s_{\delta}^{\prime}(\tau) \leq 0 \forall \tau$ due to Def. 1-iii), we get

$$
\int_{0}^{|x|^{2}} \mathrm{~s}_{\delta}(\tau) d \tau=-\int_{0}^{|x|^{2}} \tau \mathrm{s}_{\delta}^{\prime}(\tau) d \tau+|x|^{2} \mathrm{~s}_{\delta}\left(|x|^{2}\right) \geq|x|^{2} \mathrm{~s}_{\delta}\left(|x|^{2}\right)
$$

From (1), $\mathrm{s}_{\delta}\left(|x|^{2}\right) \leq \sqrt{\mathrm{s}_{\delta}\left(|x|^{2}\right)}$. Therefore, from (46),

$$
V \geq k_{1}|x|^{2} \mathrm{~s}_{\delta}\left(|x|^{2}\right)-\left(\frac{2 k_{1}}{k_{2}}\left|M_{a}\right|_{s}+\kappa\right) \sqrt{\mathrm{s}_{\delta}\left(|x|^{2}\right)}|x||y|+|M|_{i}|y|^{2}
$$

Therefore, $V$ is positive definite provided that

$$
b^{2}<4 k_{1}|M|_{i}, \quad b:=\frac{2 k_{1}}{k_{2}}\left|M_{a}\right|_{s}+\kappa
$$

which is equivalent to $\kappa<\kappa_{1}$. Since $\mathrm{s}_{\delta}(\tau)>0$ for $\tau \neq 0$ this ensures that $V$ is a positive definite function of $x$ and $y$, and $V$ is proper due to (1). Let us now prove that $V$ is non-increasing along the solutions of System (45). Differentiating $V$ along these solutions yields

$$
\left\{\begin{aligned}
\dot{V}= & -2 k_{2} y^{T} M_{s} y-2 \frac{k_{1}}{k_{2}} y^{T} M_{a} F_{\delta}(x) M y \\
& -\kappa k_{1} \mathrm{~s}_{\delta}^{2}\left(|x|^{2}\right)|x|^{2}+\kappa y^{T} F_{\delta}(x) M y \\
& -\kappa k_{2} \mathrm{~s}_{\delta}\left(|x|^{2}\right) x^{T} y \\
F_{\delta}(x):= & \mathrm{s}_{\delta}\left(|x|^{2}\right) I_{3}+2 \mathrm{~s}_{\delta}^{\prime}\left(|x|^{2}\right) x x^{T}
\end{aligned}\right.
$$

By (2), $\left|F_{\delta}(x)\right| \leq C_{\delta}, \forall x$ and we deduce that

$$
\begin{aligned}
\dot{V} & \leq-C_{1,1}|y|^{2}+C_{1,2}|y| \mathrm{s}_{\delta}\left(|x|^{2}\right)|x|-C_{1,3} \mathrm{~s}_{\delta}^{2}\left(|x|^{2}\right)|x|^{2} \\
& \leq-C_{1,1}|y|^{2}+C_{1,2}|y|\left|\operatorname{sat}_{\delta}(x)\right|-C_{1,3}\left|\operatorname{sat}_{\delta}(x)\right|^{2}
\end{aligned}
$$

with

$$
C_{1,1}:=2 k_{2}|M|_{i}-b|M|_{s} C_{\delta}, C_{1,2}:=\kappa k_{2}, C_{1,3}:=\kappa k_{1}
$$

The right-hand side of (50) is a quadratic form in $|y|$ and $\left|\operatorname{sat}_{\delta}(x)\right|$. Therefore, $\dot{V}$ is negative definite provided that

$$
\text { a) } C_{1,1}>0, \quad \text { b) } C_{1,3}>0, \quad \text { c) } C_{1,2}^{2}<4 C_{1,1} C_{1,3}
$$

Condition a) follows from the fact that $\kappa<\kappa_{2}$. Condition b) holds true for any $\kappa>0$ since $k_{1}>0$, and Condition c) follows from the fact that $\kappa<\kappa_{3}$. Thus, $\exists \beta>0$ such that

$$
\dot{V} \leq-\beta\left(|y|^{2}+\left|\operatorname{sat}_{\delta}(x)\right|^{2}\right)
$$

This shows global asymptotic stability of $(p, \dot{p})=\left(p_{r}, \dot{p}_{r}\right)$. Local exponential stability readily follows by noting that both $V$ and $\dot{V}$ are locally quadratic in $x$ and $y$ around the origin, i.e., from Def. 1-ii), $\mathrm{s}_{\delta}(\tau)=1$ for $\tau \leq \delta_{m}$.

Property ii): Since $|y|=|\tilde{v}|,(p(0), \dot{p}(0)) \in \mathcal{A}_{\rho}$ implies that $|y(0)| \leq \rho$. Applying Lemma 2 to System (42) with $\varrho \equiv 0$ and using the continuity property of the function $T$ in Lemma 2, one deduces that for any $t \geq T_{\rho}:=\max _{|y| \leq \rho} T(y)$ and along any solution with initial condition in $\mathcal{A}_{\rho}, \overline{\text { sat }}_{\bar{\delta}}$ desaturates and the solution satisfies

$$
\left\{\begin{array}{l}
\dot{x}=M y+\dot{M} \tilde{p} \\
\dot{y}=-k_{1} \operatorname{sat}_{\delta}(x)-k_{2} y
\end{array}\right.
$$


From (6) and (7),

$$
\forall t, \quad|M|_{i}|\tilde{p}(t)| \leq|x(t)|=|M(t) \tilde{p}(t)| \leq|M|_{s}|\tilde{p}(t)|
$$

Therefore, $|(x(0), y(0))| \leq \rho \max \left(1,|M|_{s}\right)$ for $(p(0), v(0)) \in \mathcal{A}_{\rho}$. Assumption A1, (54), and (55) imply that $|(\dot{x}, \dot{y})| \leq k|(x, y)|$ for some constant $k$. As a consequence, there exists a constant $\bar{\rho}$ such that, along any solution with initial condition in $\mathcal{A}_{\rho},\left|\left(x\left(T_{\rho}\right), y\left(T_{\rho}\right)\right)\right| \leq \bar{\rho}$.

Consider $V$ defined by (46) as a CLF for System (54). Its derivative along the solutions of this system satisfies

$$
\begin{aligned}
\dot{V}= & \left.\dot{V}\right|_{\text {Case } i)}+2 k_{1} \mathrm{~s}_{\delta}\left(|x|^{2}\right) x^{T} \dot{M} \tilde{p} \\
& +2 \frac{k_{1}}{k_{2}} \tilde{p}^{T} \dot{M}^{T} F_{\delta}(x) M_{a} y+\kappa \tilde{p}^{T} \dot{M}^{T} F_{\delta}(x) y \\
& +y^{T} \dot{M} y+2 \frac{k_{1}}{k_{2}} \mathrm{~s}_{\delta}\left(|x|^{2}\right) x^{T} \dot{M}_{a} y
\end{aligned}
$$

with $F_{\delta}$ given by (49) and $\left.\dot{V}\right|_{\text {Case } i)}$ the expression (49) of $\dot{V}$. Using (55) then implies that for any $M$ with $|\dot{M}|_{s}<\vartheta$,

$$
\begin{aligned}
\dot{V} \leq & \left.\dot{V}\right|_{\text {Case } i)}+\vartheta\left[\frac{2 k_{1}}{|M|_{i}} \mathrm{~s}_{\delta}\left(|x|^{2}\right)|x|^{2}+|y|^{2}\right. \\
& \left.+\left(\left(2 \frac{k_{1}}{k_{2}}\left|M_{a}\right|_{s}+\kappa\right) \frac{C_{\delta}}{|M|_{i}}+2 \frac{k_{1}}{k_{2}} \mathrm{~s}_{\delta}\left(|x|^{2}\right)\right)|x||y|\right] \\
\leq & -\left(C_{1,1}-\vartheta\right)|y|^{2}-C_{1,3}\left|\mathrm{sat}_{\delta}(x)\right|^{2} \\
& +\left(C_{1,2}+2 \frac{k_{1}}{k_{2}} \vartheta\right)\left|\operatorname{sat}_{\delta}(x)\right||y| \\
& +\frac{2 k_{1}}{|M|_{i}} \vartheta\left|x\left\|\operatorname{sat}_{\delta}(x)\left|+\left(2 \frac{k_{1}}{k_{2}}\left|M_{a}\right|_{s}+\kappa\right) \frac{C_{\delta}}{|M|_{i}} \vartheta\right| x\right\| y\right|
\end{aligned}
$$

Let:

$$
\begin{aligned}
& V_{M}:=\max _{|(x, y)| \leq \bar{\rho}} V(x, y)>0 \\
& x_{M}:=\max _{(x, y) / V(x, y) \leq V_{M}}|x|>0 \\
& s_{m}:=\min _{|x| \leq x_{M}} \mathrm{~s}_{\delta}\left(|x|^{2}\right)>0
\end{aligned}
$$

$V_{M}$ exists because $V$ is continuous and $V_{M}>0$ because $V$ is positive definite. $x_{M}$ exists because $V$ is radially unbounded and $x_{M}>0$ because $V_{M}>0$. Finally, $s_{m}$ exists because $\mathrm{s}_{\delta}$ is continuous and $s_{m}>0$ because otherwise $\mathrm{s}_{\delta}$ vanishes at some point, which contradicts $i$ ) of Definition 1. From the definition of $s_{m}$, note that

$$
|x| \leq x_{M} \Longrightarrow|x|=\left|\frac{\mathrm{s}_{\delta}\left(|x|^{2}\right) x}{\mathrm{~s}_{\delta}\left(|x|^{2}\right)}\right| \leq \frac{1}{s_{m}}\left|\operatorname{sat}_{\delta}(x)\right|
$$

Therefore, as long as $|x| \leq x_{M}$, it follows from (57) that

$$
\dot{V} \leq-C_{2,1}|y|^{2}+C_{2,2}|y|\left|\operatorname{sat}_{\delta}(x)\right|-C_{2,3}\left|\operatorname{sat}_{\delta}(x)\right|^{2}
$$

with

$$
\begin{cases}C_{2,1} & :=C_{1,1}-\vartheta \\ C_{2,2} & :=C_{1,2}+\left[2 \frac{k_{1}}{k_{2}}+\left(2 \frac{k_{1}}{k_{2}}\left|M_{a}\right|_{s}+\kappa\right) \frac{C_{\delta}}{s_{m}|M|_{i}}\right] \vartheta \\ C_{2,3} & :=C_{1,3}-\frac{2 k_{1}}{s_{m}|M|_{i}} \vartheta\end{cases}
$$

From the above expression, the $C_{2, j}$ 's tend to the $C_{1, j}$ 's as $\vartheta$ tends to zero. Thus, it follows from (52) that for $\vartheta>0$ small enough and $\kappa$ satisfying (47), the right-hand side of (60) is a negative-definite quadratic form in $|y|$ and $\left|\operatorname{sat}_{\delta}(x)\right|$. We thus have shown that for $\vartheta>0$ small enough, $0 \neq|x| \leq x_{M} \Longrightarrow$ $\dot{V}<0$. From the definition of $x_{M}$, we deduce that $0 \neq V \leq$ $V_{M} \Longrightarrow \dot{V}<0$. From the definition of $V_{M}$ and the fact that $\left|\left(x\left(T_{\rho}\right), y\left(T_{\rho}\right)\right)\right| \leq \bar{\rho}$ along any solution with initial condition in
$\mathcal{A}_{\rho}$, this implies convergence of $(x, y)$ to zero along these solutions. Local exponential stability is proved as for Property $i$ ).

Proof of Proposition 2: It builds on the proof of Prop. 1. Recall from Prop. 1 that $x=M \tilde{p}$ and $y=\dot{\tilde{p}}$. In addition, let $z:=R \eta$ and $\tilde{\mu}:=\mu-\mu_{3} e_{3}$. Using the expression (15) of $\ddot{p}$, the expression of $u$ in (19), and (18), one obtains (compare with (42)):

$$
\left\{\begin{aligned}
\dot{x} & =M y+\dot{M} \tilde{p} \\
\dot{y} & =-k_{1} \operatorname{sat}_{\delta}(z)-k_{2} \overline{\operatorname{sat}}_{\bar{\delta}}(y)+R \tilde{\mu} \\
\dot{z} & =-k_{3}(z-x)
\end{aligned}\right.
$$

Lemma 3 With $\omega_{1}, \omega_{2}$ defined by (19), $\tilde{\mu}=0$ is LES. More precisely, $\exists \bar{c}, \alpha>0:|\tilde{\mu}(t)| \leq \bar{c}|\tilde{\mu}(0)| e^{-\alpha t}, \forall t$, for any initial condition such that $\mu(0) \neq-|\mu(0)| e_{3}$.

Property i): Assumption A2 implies that $\dot{M}=0$. From Lemma $3 \tilde{\mu}$ tends to zero and from (12) $k_{1} \delta_{M}-k_{2} \bar{\delta}_{m}<0$. Then, Lemma 2 applies to the second equation in (62) and along any solution, $\overline{\text { sat }}_{\bar{\delta}}$ desaturates after some time and the solution then satisfies:

$$
\begin{cases}\dot{x}= & M y \\ \dot{y} & =-k_{1} \operatorname{sat}_{\delta}(x)-k_{2} y \\ & -k_{1}\left[\operatorname{sat}_{\delta}(z)-\operatorname{sat}_{\delta}(x)\right]+R \tilde{\mu} \\ \dot{z}-\dot{x}= & -k_{3}(z-x)-M y\end{cases}
$$

Note that System (63) is equivalent to System (45) when $z=x$ and $\tilde{\mu}=0$. Consider the CLF $V_{1}$ defined by

$$
V_{1}(x, y, z)=V(x, y)+(z-x)^{2}
$$

We deduce from the mean-value inequality and (2) that $\mid \operatorname{sat}_{\delta}(z)-$ sat $_{\delta}(x)\left|\leq C_{\delta}\right| z-x \mid \forall(z, x)$. Therefore, the time-derivative $\dot{V}_{1}$ of $V_{1}$ along the solutions of (63) satisfies:

$$
\left\{\begin{aligned}
& \dot{V}_{1} \leq-C_{3,1}|y|^{2}-C_{3,2}|z-x|^{2}-C_{3,3} \mathrm{~s}_{\delta}^{2}\left(|x|^{2}\right)|x|^{2} \\
&+C_{3,4}|y||z-x|+C_{3,5}|y| \mathrm{s}_{\delta}\left(|x|^{2}\right)|x| \\
&+C_{3,6}|z-x| \mathrm{s}_{\delta}\left(|x|^{2}\right)|x|+\frac{\partial V}{\partial y} R \tilde{\mu} \\
& C_{3,1}::=C_{1,1}, C_{3,2}:=2 k_{3}, C_{3,3}:=C_{1,3} \\
& C_{3,4}:=2\left(C_{\delta} k_{1}+1\right)\left|M_{s}\right|_{s}, C_{3,5}:=C_{1,2}, C_{3,6}:=b C_{\delta} k_{1}
\end{aligned}\right.
$$

with the $C_{1, j}$ 's given by (51).

Let us first assume that $\tilde{\mu}=0$. We claim that $V_{1}$ is a Lyapunov function for a proper choice of $\kappa$ and $k_{3}$. Let $0<\kappa<$ $\min \left\{\kappa_{1}, \kappa_{2}\right\}$ with $\kappa_{1}, \kappa_{2}$ defined by (47). From the proof of Prop. $1, \kappa<\kappa_{1}$ implies that $V$ is positive definite and proper, so that $V_{1}$ is positive definite and proper too. Then, $0<\kappa<\kappa_{2}$ implies that $C_{3,1}>0$. Since $C_{3,2}, C_{3,3}>0$ and $\dot{V}_{1}$ is a quadratic form in $|y|,|z-x|$, and $\mathrm{s}_{\delta}^{2}\left(|x|^{2}\right)|x|=\left|\operatorname{sat}_{\delta}(x)\right|, \dot{V}_{1}$ is negative definite provided that

$$
\begin{aligned}
4 C_{3,1} C_{3,2} C_{3,3} & >C_{3,1} C_{3,6}^{2}+C_{3,2} C_{3,5}^{2}+C_{3,3} C_{3,4}^{2} \\
& +\frac{1}{2} C_{3,4} C_{3,5} C_{3,6}
\end{aligned}
$$

This condition is satisfied by a proper choice of $\kappa$ and $k_{3}$. Indeed, the only term depending on $k_{3}$ in (66) is $C_{3,2}$. Thus, (66) is satisfied for $k_{3}$ large enough provided that $4 C_{3,1} C_{3,3}>C_{3,5}^{2}$. 
From (65), this is equivalent to $4 C_{1,1} C_{1,3}>C_{1,2}^{2}$. This inequality, which corresponds to Condition c) in (52), is satisfied for $\kappa>0$ small enough.

Let us now take into account the additive perturbation $\tilde{\mu}$. It follows from (65) and (66) that for some $\beta>0$,

$$
\dot{V}_{1} \leq-\beta\left(|y|^{2}+|z-x|^{2}+\left|\operatorname{sat}_{\delta}(x)\right|^{2}\right)+\left|\frac{\partial V}{\partial y} \| \tilde{\mu}\right|
$$

and from (46), there exists $c>0$ such that

$$
\left|\frac{\partial V}{\partial y}(x, y)\right| \leq c\left|\left(y, \operatorname{sat}_{\delta}(x)\right)\right| \quad \forall(x, y)
$$

Therefore, by the triangular inequality,

$$
\dot{V}_{1} \leq-\frac{\beta}{2}\left(|y|^{2}+|z-x|^{2}+\left|\operatorname{sat}_{\delta}(x)\right|^{2}\right)+\frac{c^{2}|\tilde{\mu}|^{2}}{2 \beta}
$$

Convergence to zero of $V_{1}$ then follows from the convergence of $\tilde{\mu}$ to zero. This, together with Lemma 3 implies the convergence of $(p, \dot{p}, q, \eta)$ to $\left(p_{r}, \dot{p}_{r}, q_{r}, 0\right)$ from any initial condition in $\mathcal{A}$. Finally, local exponential stability follows from local exponential stability of $\tilde{\mu}=0$ (Lemma 3 ) and the fact that saturation functions are identity functions around the origin (i.e., System (62) is locally linear).

Property ii): Lemma 3 still implies that $\tilde{\mu}$ exponentially converges to zero. Proceeding as in the proof of Prop. 1-ii), one deduces from Lemma 2 that for any $t \geq T_{\rho}:=\max _{|y| \leq \rho} T(y)$ and along any solution starting from $\mathcal{A}_{\rho}, \overline{\text { sat }}_{\bar{\delta}}$ desaturates and the solution satisfies

$$
\left\{\begin{array}{lll}
\dot{x} & =M y+\dot{M} \tilde{p} \\
\dot{y} & =-k_{1} \operatorname{sat}_{\delta}(x)-k_{2} y \\
& -k_{1}\left[\operatorname{sat}_{\delta}(z)-\operatorname{sat}_{\delta}(x)\right]+R \tilde{\mu} \\
\dot{z}-\dot{x}= & -k_{3}(z-x)-M y-\dot{M} \tilde{p}
\end{array}\right.
$$

Note that System (69) is equivalent to System (54) when $z=x$ and $\tilde{\mu}=0$. Since $\tilde{\mu}$ exponentially converges to zero and $|\tilde{\mu}(0)| \leq$ $|\mu(0)| \leq 2 g+k_{1} \delta_{M}+k_{2} \bar{\delta}_{M}$, there exists $T_{1}>0$ such that, for any initial condition in $\mathcal{A}_{\rho}$,

$$
|\tilde{\mu}(t)| \leq \frac{\beta \delta_{m}}{c \sqrt{6}}, \quad \forall t \geq T_{1}
$$

with $\beta$ satisfying (67). Thus, for $t \geq \bar{T}_{\rho}:=\max \left\{T_{\rho}, T_{1}\right\}$, both (69) and (70) are satisfied. Proceeding as in the proof of Prop. 1-ii), one also deduces from (55) that for some $\bar{\rho}$ and along any solution with initial condition in $\mathcal{A}_{\rho}, \mid\left(x\left(\bar{T}_{\rho}\right), y\left(\bar{T}_{\rho}\right),(z-\right.$ $\left.x)\left(\bar{T}_{\rho}\right)\right) \mid \leq \rho_{1}$. Let $\bar{\rho}:=\max \left\{\rho_{1}, \delta_{m}\right\}$ so that $\mid\left(x\left(\bar{T}_{\rho}\right), y\left(\bar{T}_{\rho}\right),(z-\right.$ $\left.x)\left(\bar{T}_{\rho}\right)\right) \mid \leq \bar{\rho}$. We consider again the CLF $V_{1}$ defined by (64) and define $s_{m}, x_{M}, V_{M}$ as follows (compare with (58)):

$$
\begin{aligned}
& V_{M}:=\max _{|(x, y, z-x)| \leq \bar{\rho}} V_{1}(x, y, z)>0 \\
& x_{M}:=\max _{V_{1}(x, y, z) \leq V_{M}}|x|>0 \\
& s_{m}:=\min _{|x| \leq x_{M}} \mathrm{~s}_{\delta}\left(|x|^{2}\right)>0
\end{aligned}
$$

By using (59), one deduces from (69) that for $|x| \leq x_{M}$ and for $M$ such that $|\dot{M}|_{s}<\vartheta$,

$$
\begin{aligned}
\dot{V}_{1} \leq & -C_{4,1}|y|^{2}-C_{4,2}|z-x|^{2}-C_{4,3} \operatorname{sat}_{\delta}^{2}\left(|x|^{2}\right)|x|^{2} \\
& +C_{4,4}|y \| z-x|+C_{4,5}|y| \operatorname{sat}_{\delta}\left(|x|^{2}\right)|x| \\
& +C_{4,6}|z-x| \mathrm{sat}_{\delta}\left(|x|^{2}\right)|x|+\frac{\partial V}{\partial y} R \tilde{\mu}
\end{aligned}
$$

with:

$$
\left\{\begin{array}{l}
C_{4,1}:=C_{3,1}-\vartheta, C_{4,2}:=C_{3,2} \\
C_{4,3}:=C_{3,3}-\frac{2 k_{1}}{s_{m}|M|_{i}} \vartheta, C_{4,4}:=C_{3,4} \\
C_{4,5}:=C_{3,5}+\left[2 \frac{k_{1}}{k_{2}}+\left(2 \frac{k_{1}}{k_{2}}\left|M_{a}\right|_{s}+\kappa\right) \frac{C_{\delta}}{s_{m}|M|_{i}}\right] \vartheta \\
C_{4,6}:=C_{3,6}+\frac{\vartheta}{s_{m}|M|_{i}}
\end{array}\right.
$$

Let us choose $\kappa$ and $k_{3}$ as in the proof of case $i$ ) above, so that (66) is satisfied. Since the $C_{4, j}$ 's tend to the $C_{3, j}$ 's as $\vartheta$ tends to zero, for $\vartheta>0$ small enough the following inequality is satisfied (compare with (67)):

$$
\dot{V}_{1} \leq-\frac{2 \beta}{3}\left(|y|^{2}+|z-x|^{2}+\left|\operatorname{sat}_{\delta}(x)\right|^{2}\right)+\left|\frac{\partial V}{\partial y} \| \tilde{\mu}\right|
$$

Therefore, by (68) and the triangular inequality,

$$
\dot{V}_{1} \leq-\frac{\beta}{6}\left(|y|^{2}+|z-x|^{2}+\left|\operatorname{sat}_{\delta}(x)\right|^{2}\right)+\frac{c^{2}|\tilde{\mu}|^{2}}{2 \beta}
$$

Recall that this relation is true as long as $|x(t)| \leq x_{M}$ and $t \geq$ $\bar{T}_{\rho}$. In particular, it is true at $t=\bar{T}_{\rho}$ because $\mid\left(x\left(\bar{T}_{\rho}\right), y\left(\bar{T}_{\rho}\right),(z-\right.$ $\left.x)\left(\bar{T}_{\rho}\right)\right) \mid \leq \bar{\rho}$ (see above) and from (71),

$$
|(x, y, z-x)| \leq \bar{\rho} \Longrightarrow V_{1}(x, y, z) \leq V_{M} \Longrightarrow|x| \leq x_{M}
$$

From (73) and (70), $|x(t)| \leq x_{M}$ and $t \geq \bar{T}_{\rho}$ imply that

$$
\dot{V}_{1} \leq-\frac{\beta}{6}\left(|y|^{2}+|z-x|^{2}+\left|\operatorname{sat}_{\delta}(x)\right|^{2}-\frac{\delta_{m}^{2}}{2}\right)
$$

We claim that

$$
V_{1}(x, y, z)=V_{M} \Longrightarrow|(x, y, z-x)| \geq \delta_{m}
$$

Indeed, otherwise, from the definition of $V_{M}$ in (71) and the fact that $\bar{\rho} \geq \delta_{m}$, on the set $\{(x, y, z):|(x, y, z-x)| \leq \bar{\rho}\} V_{1}$ reaches its maximum in the interior of this set, which implies that $V_{1}$ has a critical point. This contradicts (72) that implies $V_{1}$ is a Lyapunov function for $\tilde{\mu}=0$.

From (71), $V_{1}=V_{M}$ implies that $|x| \leq x_{M}$ and thus, (74) holds true. We thus deduce from (74), (75), and the properties of the function $\mathrm{sat}_{\delta}$ that for any $t \geq \bar{T}_{\rho}, V_{1}=V_{M} \Longrightarrow \dot{V}_{1} \leq$ $-\frac{\beta}{6} \frac{\delta_{m}^{2}}{2}<0$. Since $V_{1} \leq V_{M}$ at $t=\bar{T}_{\rho}, V_{1} \leq V_{M}$ everafter and thus, $|x| \leq x_{M}$ everafter. Then, (73) is satisfied for any $t \geq \bar{T}_{\rho}$ and convergence to zero of $x, y, z$ follows from the convergence of $\tilde{\mu}$ to zero. Local exponential stability is deduced as for Property $i$ ).

Proof of Proposition 3: Let $x=R \tilde{\sigma}, y=R \tilde{v}, Y=\left(\mu_{1}, \mu_{2}\right)^{T}$, and $\varepsilon=\frac{1}{k_{4}}$. One obtains in closed-loop, after some calculations:

$$
\left\{\begin{aligned}
\dot{x}= & M y+\dot{M} \tilde{p} \\
\dot{y}= & -k_{1} \operatorname{sat}_{\delta}(x)-k_{2} \overline{\operatorname{sat}}_{\bar{\delta}}(y)+R\left(Y_{1}, Y_{2}, 0\right)^{T} \\
\varepsilon \dot{Y}= & -\mu_{3} Y+\varepsilon \omega_{3}(t) Y^{\perp} \\
& +\varepsilon R_{1,2}^{T}\left(k_{1} F_{\delta}(x) \dot{x}+k_{2} \bar{F}_{\bar{\delta}}(y) \dot{y}\right)
\end{aligned}\right.
$$

with $Y^{\perp}=\left(Y_{2},-Y_{1}\right)^{T}, R_{1,2}^{T}$ the first two lines of $R^{T}$, and

$$
\begin{cases}F_{\delta}(x) & :=\mathrm{s}_{\delta}\left(|x|^{2}\right) I+2 \mathrm{~s}_{\delta}^{\prime}\left(|x|^{2}\right) x x^{T} \\ \bar{F}_{\bar{\delta}}(y) & :=\overline{\mathrm{s}}_{\bar{\delta}}\left(|y|^{2}\right) I+2 \overline{\mathrm{s}}_{\bar{\delta}}^{\prime}\left(|y|^{2}\right) y y^{T}\end{cases}
$$

Property i): It relies on the following lemma. 
Lemma 4 Assume A1, A2, and A3. Then, for any $\rho>0$ there exists $\varepsilon_{\rho}, T>0$ such that, for any $\varepsilon \in\left(0, \varepsilon_{\rho}\right)$, any $t \geq T$, and along any solution of the system with initial condition in $\mathcal{A}_{\rho}$ :

a) $|y(t)| \leq \bar{\delta}_{m}$ (i.e. the function $\overline{\mathrm{sat}}_{\bar{\delta}}$ desaturates);

b) $\mu_{3}(t)>g \varkappa / \sqrt{2}$.

Lemma 4 implies that by choosing $\varepsilon$ small enough, along any trajectory with initial condition in $\mathcal{A}_{\rho}$, the function $\overline{\mathrm{sat}}_{\bar{\delta}}$ desaturates after some time, so that the trajectory becomes solution to the system

$$
\left\{\begin{aligned}
\dot{x}= & M y \\
\dot{y}= & -k_{1} \operatorname{sat}_{\delta}(x)-k_{2} y+R\left(Y_{1}, Y_{2}, 0\right)^{T} \\
\varepsilon \dot{Y}= & -\mu_{3} Y+\varepsilon \omega_{3}(t) Y^{\perp} \\
& +\varepsilon R_{1,2}^{T}\left(k_{1} F_{\delta}(x) \dot{x}+k_{2} \bar{F}_{\bar{\delta}}(y) \dot{y}\right)
\end{aligned}\right.
$$

The first two equations of this system correspond to (45), modulo the additional term $R\left(Y_{1}, Y_{2}, 0\right)^{T}$. Then, using (53), property b) of Lemma 4, and (68), one deduces that $V_{2}(x, y, Y):=$ $V(x, y)+|Y|^{2}$ is a Lyapunov function for System (78) for $\varepsilon$ small enough, with $\dot{V}$ upper-bounded by a negative definite quadratic function of $\operatorname{sat}_{\delta}(x), y$, and $Y$.

Property ii): It relies on the following lemma.

Lemma 5 Assume A1 and A2. Then, for any $\rho>0$ there exist $\varepsilon_{\rho}, T_{0}, \bar{c}_{5}, \beta, c, \vartheta>0$ such that, for any $\varepsilon \in\left(0, \varepsilon_{\rho}\right)$, any $t \geq T_{0}$, any $M$ such that $|\dot{M}|_{s}<\vartheta$, and along any solution of the system with initial condition in $\mathcal{A}_{\rho}$ :

a) $|x(t)| \leq \bar{c}_{5}$;

b) $|y(t)| \leq \bar{\delta}_{m}$ (i.e. the function $\overline{\mathrm{sat}}_{\bar{\delta}}$ desaturates);

c) $\mu_{3}(t)>g \varkappa / \sqrt{2}$; (46).

d) $\dot{V} \leq-\frac{\beta}{2}\left|\left(y, \operatorname{sat}_{\delta}(x)\right)\right|^{2}+c \mid\left(y\right.$, sat $\left._{\delta}(x)\right)|| Y \mid$, with $V$ defined by

Lemma 5 implies that by choosing $\varepsilon$ and $\vartheta$ small enough, along any trajectory with initial condition in $\mathcal{A}_{\rho}$, the function $\overline{\mathrm{sat}}_{\bar{\delta}}$ desaturates after time $T_{0}$, so that the trajectory becomes solution to the system

$$
\left\{\begin{aligned}
\dot{x}= & M y+\dot{M} \tilde{p} \\
\dot{y}= & -k_{1} \operatorname{sat}_{\delta}(x)-k_{2} y+R\left(Y_{1}, Y_{2}, 0\right)^{T} \\
\varepsilon \dot{Y}= & -\mu_{3} Y+\varepsilon \omega_{3}(t) Y^{\perp} \\
& +\varepsilon R_{1,2}^{T}\left(k_{1} F_{\delta}(x) \dot{x}+k_{2} \bar{F}_{\bar{\delta}}(y) \dot{y}\right)
\end{aligned}\right.
$$

The first two equations of this system correspond to (54), modulo the term $R\left(Y_{1}, Y_{2}, 0\right)^{T}$. Property a) of Lemma 5 implies that $x$ is bounded, so that $|(\dot{x}, \dot{y})|$ is bounded by a linear function of $|y|,\left|\operatorname{sat}_{\delta}(x)\right|$, and $|Y|$ (see (59)). From here, Properties c) and d) of Lemma 4 and boundedness of $F_{\delta}, \bar{F}_{\bar{\delta}}$ imply that $V_{2}(x, y, Y):=V(x, y)+|Y|^{2}$ is a Lyapunov function for System (79) for $\varepsilon$ small enough.

Proof of Proposition 4: Let $\bar{e}_{\sigma}:=\operatorname{Re}_{\sigma}=R \hat{\sigma}-M \tilde{p}, \bar{e}_{v}:=$ $R e_{v}=R \hat{v}_{M}-M \dot{\tilde{p}}$. From (30)-(31), $\dot{\bar{e}}_{\sigma}=\bar{e}_{v}-2 \alpha k \bar{e}_{\sigma}$ and $\dot{\bar{e}}_{v}=$ $-k^{2} \bar{e}_{\sigma}-M \ddot{p}$. Thus, $e_{\sigma}$ is solution to a Hurwitz-stable second order linear equation with bounded input $u_{b}:=-M \ddot{p}$, i.e., $\ddot{\bar{e}}_{\sigma}+$ $2 \alpha k \dot{\bar{e}}_{\sigma}+k^{2} \bar{e}_{\sigma}=u_{b}$. Let $\tau:=k t$ and ' denote the derivative w.r.t. $\tau$. Then, the previous equation becomes $\bar{e}_{\sigma}^{\prime \prime}+2 \alpha \bar{e}_{\sigma}^{\prime}+\bar{e}_{\sigma}=\frac{u_{b}}{k^{2}}$. Since $u_{b}$ is bounded, this implies the ultimate boundedness of $\left|\bar{e}_{\sigma}\right|$ and $\left|\bar{e}_{\sigma}^{\prime}\right|$ by a value proportional to $1 / k^{2}$. Hence, $\left|\bar{e}_{\sigma}\right|$ and $\left|\dot{\bar{e}}_{\sigma}\right|$ are ultimately bounded by a value proportional to $1 / k^{2}$ and $1 / k$ respectively. The same property holds for $\left|e_{\sigma}\right|$ and $\left|e_{v}\right|$.

Proof of Proposition 5: Let $x:=R \tilde{\sigma}=M \tilde{p}$ and $y:=M^{-1} R v_{M}=$ $\dot{p}$. Then, $x, y$ satisfy the following equations:

$$
\left\{\begin{aligned}
\dot{x} & =M y \\
\dot{y} & =-k_{1} \operatorname{sat}_{\delta}(x)-k_{2} \overline{\operatorname{sat}}_{\bar{\delta}}(M y)
\end{aligned}\right.
$$

This is the same as (42), except for the presence of the matrix $M$ in the term $\overline{\operatorname{sat}}_{\bar{\delta}}(M y)$. This similitude allows one to duplicate the proof of Prop. 1 modulo minor adaptations detailed below.

Lemma 2, with $\overline{\operatorname{sat}}_{\bar{\delta}}(y)$ in (43) replaced by $\overline{\operatorname{sat}}_{\bar{\delta}}(M y)$ and the desaturation condition (44) replaced by

$$
|M \varrho(t)| \leq k_{2} \bar{\delta}_{m}|M|_{i}-k_{1} \delta_{M}|M|_{s}-c_{\varrho}, \quad \forall t \geq t_{1}
$$

still holds true to show that $\overline{\operatorname{sat}}_{\bar{\delta}}(M y)$ desaturates. Indeed, $\bar{y}:=$ My satisfies the equation

$$
\dot{\bar{y}}=-k_{1} M \operatorname{sat}_{\delta}(x)-k_{2} M \overline{\operatorname{sat}}_{\bar{\delta}}(\bar{y})+M \varrho(t)
$$

and the proof proceeds like for Lemma 2, by considering the Lyapunov function $V_{0}$ defined by $V_{0}(\bar{y})=\frac{1}{2}|\bar{y}|^{2}$. The second inequality in (32) ensures that (81) is satisfied with $\varrho \equiv 0$ and some $c_{\varrho}>0$. After desaturation, solutions to (80) satisfy the following equations (compare with (45)):

$$
\left\{\begin{array}{l}
\dot{x}=M y \\
\dot{y}=-k_{1} \operatorname{sat}_{\delta}(x)-k_{2} M y
\end{array}\right.
$$

The Lyapunov function in (46) is modified as follows:

$$
\begin{aligned}
V(x, y)= & k_{1} \int_{0}^{|x|^{2}} \mathrm{~s}_{\delta}(\tau) d \tau+y^{T} M y \\
& +\frac{2 k_{1}}{k_{2}} \operatorname{sat}_{\delta}(x)^{T} M_{a} M^{-1} y+\kappa \operatorname{sat}_{\delta}(x)^{T} y
\end{aligned}
$$

i.e., $V$ so defined only differs from (46) by the matrix term $M_{a} M^{-1}$ (in place of $M_{a}$ ). We show that $V$ is a Lyapunov function for $\kappa$ small enough. Let (compare with (47))

$$
\left\{\begin{aligned}
\bar{\kappa} & :=k_{2}^{2}|M|_{i}^{2}-k_{1}\left|M_{a} M^{-1}\right|_{s}|M|_{s} C_{\delta}>0 \\
\kappa_{1} & :=2 \sqrt{k_{1}|M|_{i}}-2 \frac{k_{1}}{k_{2}}\left|M_{a} M^{-1}\right|_{s}>0 \\
\kappa_{2} & :=\frac{2 \bar{k}}{k_{2}|M|_{s} C_{\delta}}>0 \\
\kappa_{3} & :=\frac{k_{2}}{k_{2}|M|_{s} C_{\delta}+\frac{k_{2}^{3}|M|_{s}^{2}}{4 k_{1}}}>0 \\
0<\kappa & <\min \left\{\kappa_{1}, \kappa_{2}, \kappa_{3}\right\}
\end{aligned}\right.
$$

where positivity of $\bar{\kappa}, \kappa_{1}$ follows from (32) and positivity of $\kappa_{2}, \kappa_{3}$ is a consequence thereof. Positive definiteness of $V$ is still ensured by (48), with $b$ now defined by $b:=\frac{2 k_{1}}{k_{2}}\left|M_{a} M^{-1}\right|_{s}+\kappa$. This yields the condition $\kappa<\kappa_{1}$. Differentiating $V$ along the solutions of System (82) yields

$$
\left\{\begin{aligned}
\dot{V} & =-2 k_{2} y^{T} M_{s} M y+2 \frac{k_{1}}{k_{2}} y^{T}\left(M_{a} M^{-1}\right)^{T} F_{\delta}(x) M y \\
& -\kappa k_{1} \mathrm{~s}_{\delta}^{2}\left(|x|^{2}\right)|x|^{2}+\kappa y^{T} F_{\delta}(x) M y \\
& -\kappa k_{2} \mathrm{~s}_{\delta}\left(|x|^{2}\right) x^{T} M y-2 \frac{k_{1}^{2}}{k_{2}} \operatorname{sat}_{\delta}(x)^{T} M_{a} M^{-1} \operatorname{sat}_{\delta}(x)
\end{aligned}\right.
$$

We now use the assumption that $M_{a}$ and $M_{s}$ commute. This implies that, for any $\xi \in \mathbb{R}^{3}, \xi^{T} M_{s} M_{a} \xi=0$ and

$$
\xi^{T} M_{a} M^{-1} \xi=\left(\left(M_{a}+M_{s}\right) M^{-1} \xi\right)^{T} M_{a} M^{-1} \xi=\left|M_{a} M^{-1} \xi\right|^{2}
$$


Therefore, one deduces from (85) that

$$
\left\{\begin{array}{c}
\dot{V} \leq-2 k_{2}\left|M_{s} y\right|^{2}+2 \frac{k_{1}}{k_{2}} y^{T}\left(M_{a} M^{-1}\right)^{T} F_{\delta}(x) M y \\
\quad-\kappa k_{1} \mathrm{~s}_{\delta}^{2}\left(|x|^{2}\right)|x|^{2}+\kappa y^{T} F_{\delta}(x) M y \\
\quad-\kappa k_{2} \mathrm{~s}_{\delta}\left(|x|^{2}\right) x^{T} M y
\end{array}\right.
$$

This implies that (50) is still satisfied with the $C_{1, j}$ 's defined by (compare with (51))

$$
C_{1,1}:=2 k_{2}|M|_{i}^{2}-b|M|_{s} C_{\delta}, C_{1,2}:=\kappa k_{2}|M|_{s}, C_{1,3}:=\kappa k_{1}
$$

Thus, $\dot{V}$ is negative definite provided that (52) is satisfied with the above-defined $C_{1, j}$ 's. Condition a) follows from the fact that $\kappa<\kappa_{2}$. Condition $\mathrm{b}$ ) holds true for any $\kappa>0$ since $k_{1}>0$, and Condition c) follows from the fact that $\kappa<\kappa_{3}$. Thus, (53) is satisfied for some $\beta>0$ and the end of the proof follows like for Proposition 1.

Proof of Proposition 6: Let $x:=R \tilde{\sigma}, y:=R \tilde{v}, Y:=\left(\mu_{1}, \mu_{2}\right)^{T}$, and $\varepsilon:=\frac{1}{k_{4}}$. One obtains in closed-loop, after some calculations (compare with (76) for $\dot{M}=0$ ):

$$
\left\{\begin{aligned}
\dot{x}= & M y \\
\dot{y}= & -k_{1} \operatorname{sat}_{\delta}(x)-k_{2} \overline{\operatorname{sat}}_{\bar{\delta}}(M y)+R\left(Y_{1}, Y_{2}, 0\right)^{T} \\
\varepsilon \dot{Y}= & -\mu_{3} Y+\varepsilon \omega_{3}(t) Y^{\perp} \\
& +\varepsilon R_{1,2}^{T}\left(k_{1} F_{\delta}(x) \dot{x}+k_{2} \bar{F}_{\bar{\delta}}(M y) M \dot{y}\right)
\end{aligned}\right.
$$

with $Y^{\perp}=\left(Y_{2},-Y_{1}\right)^{T}, R_{1,2}^{T}$ the first two lines of $R^{T}$, and $F_{\delta}, \bar{F}_{\bar{\delta}}$ defined by (77). By setting, like in the proof of Prop. $5, \bar{y}:=$ $M y=R^{T} v_{M}$, the above equations can also be written as

$$
\left\{\begin{aligned}
\dot{x}= & \bar{y} \\
\dot{\bar{y}}= & -k_{1} M \operatorname{sat}_{\delta}(x)-k_{2} M \overline{\operatorname{sat}}_{\bar{\delta}}(\bar{y})+\operatorname{MR}\left(Y_{1}, Y_{2}, 0\right)^{T} \\
\varepsilon \dot{Y}= & -\mu_{3} Y+\varepsilon \omega_{3}(t) Y^{\perp} \\
& +\varepsilon R_{1,2}^{T}\left(k_{1} F_{\delta}(x) \dot{x}+k_{2} \bar{F}_{\bar{\delta}}(\bar{y}) \dot{\bar{y}}\right)
\end{aligned}\right.
$$

We claim that the conclusion of Lemma 4 is still valid with statement $a$ ) replaced by:

a) $|\bar{y}(t)| \leq \bar{\delta}_{m}$ (i.e. the function $\overline{\mathrm{sat}}_{\bar{\delta}}$ desaturates)

Indeed, based on (87) the proof follows exactly that of Lemma 4 with $y$ replaced everywhere by $\bar{y}$, and the constant $k_{2} \bar{\delta}_{m}-$ $k_{1} \delta_{M}$ in (102) and subsequent equations replaced everywhere by $k_{2} \bar{\delta}_{m}|M|_{i}-k_{1} \delta_{M}|M|_{s}$ (compare with (81)). Note also that the value of the constants $c_{1}, c_{2}$ must be changed as follows: $c_{1}:=k_{1} \delta_{M}+k_{2} \bar{\delta}_{M}+g$ (from (35)) and therefore, from (88), $c_{2}:=|M|_{s}\left(2 k_{1} \delta_{M}+2 k_{2} \bar{\delta}_{M}+g\right)$.

After desaturation of the function $\overline{\mathrm{sat}}_{\bar{\delta}}$, the trajectories of System (87) become solution to the system

$$
\left\{\begin{aligned}
\dot{x}= & M y \\
\dot{y}= & -k_{1} \operatorname{sat}_{\delta}(x)-k_{2} M y+R\left(Y_{1}, Y_{2}, 0\right)^{T} \\
\varepsilon \dot{Y}= & -\mu_{3} Y+\varepsilon \omega_{3}(t) Y^{\perp} \\
& +\varepsilon R_{1,2}^{T}\left(k_{1} F_{\delta}(x) \dot{x}+k_{2} \bar{F}_{\bar{\delta}}(M y) M \dot{y}\right)
\end{aligned}\right.
$$

The first two equations in (89) correspond to (82) modulo the additional term $R\left(Y_{1}, Y_{2}, 0\right)^{T}$. The end of proof follows as for Prop. 3, using the fact that $V$ in (83) satisfies (53).

\section{Proof of Lemmas}

Proof of Lemma 1: We proceed by contradiction. Assume that $\mu(0)=-|\mu(0)| e_{3}$. From (20) and (23) $\mu(0) \neq 0$. From (20), $\gamma(0)+|\mu(0)| e_{3}=-\left(k_{1} \operatorname{sat}_{\delta}(\eta)+k_{2} \overline{\operatorname{sat}}_{\bar{\delta}}(\tilde{v})-a_{r}\right)$. Thus,

$$
\begin{aligned}
&\left|k_{1} \operatorname{sat}_{\delta}(\eta)+k_{2} \overline{\operatorname{sat}}_{\bar{\delta}}(\tilde{v})-a_{r}\right|^{2}=|\gamma(0)+| \mu(0)\left|e_{3}\right|^{2} \\
&=|\gamma(0)|^{2}+|\mu(0)|^{2}+2|\mu(0)| \gamma_{3}(0) \\
&=g^{2}+|\mu(0)|^{2}+2|\mu(0)| \gamma_{3}(0) \\
&> g^{2}+|\mu(0)|^{2}-2|\mu(0)| \sqrt{g^{2}-\left(k_{1} \delta_{M}+k_{2} \bar{\delta}_{M}+\left|\ddot{p}_{r}\right|_{s}\right)^{2}} \\
&>\left(k_{1} \delta_{M}+k_{2} \bar{\delta}_{M}+\left|\ddot{p}_{r}\right|_{s}\right)^{2} \\
&+\left(\sqrt{g^{2}-\left(k_{1} \delta_{M}+k_{2} \bar{\delta}_{M}+\left|\ddot{p}_{r}\right|_{s}\right)^{2}}-|\mu(0)|\right)^{2} \\
&>\left(k_{1} \delta_{M}+k_{2} \bar{\delta}_{M}+\left|\ddot{p}_{r}\right|_{s}\right)^{2}
\end{aligned}
$$

where the first inequality comes from (24) and the fact that $\mu(0) \neq 0$. This contradicts (23).

Proof of Lemma 2: Consider the function $V_{0}$ defined by $V_{0}(y)=$ $\frac{1}{2}|y|^{2}$. Its derivative along the solutions of Eq. (43) is given by

$$
\begin{aligned}
\dot{V}_{0} & =-k_{1} y^{T} \operatorname{sat}_{\delta}(x)-k_{2} y^{T} \overline{\operatorname{sat}}_{\bar{\delta}}(y)+y^{T} \varrho(t) \\
& \leq k_{1}|y| \delta_{M}-k_{2} y^{T} \overline{\operatorname{sat}}_{\bar{\delta}}(y)+|y| \varrho(t) \mid
\end{aligned}
$$

Case 1: $t \in\left[0, t_{1}\right]$. Let $|\varrho|_{s}$ denote the max of $\varrho$ on this time interval. From the above inequality,

$$
\dot{V}_{0} \leq k_{1}|y| \delta_{M}+\left.|y| \varrho\right|_{s} \leq\left(k_{1} \delta_{M}+|\varrho|_{s}\right) \sqrt{2 V_{0}}
$$

The comparison lemma Khalil (2002) then yields

$$
\left|y\left(t_{1}\right)\right| \leq|y(0)|+\left(k_{1} \delta_{M}+|\varrho| s\right) t_{1}
$$

Case 2: $t \geq t_{1}$. From Def. 1,

$$
\begin{aligned}
|y| \geq \bar{\delta}_{m} \Longrightarrow y^{T} \overline{\operatorname{sat}}_{\bar{\delta}}(y) & =|y|^{2} \overline{\mathrm{S}}_{\bar{\delta}}\left(|y|^{2}\right)=|y| \overline{\mathrm{S}}_{\bar{\delta}}\left(|y|^{2}\right)|y| \\
& \geq|y| \overline{\mathbf{S}}_{\bar{\delta}}\left(\left|\bar{\delta}_{m}\right|^{2}\right) \bar{\delta}_{m} \geq|y| \bar{\delta}_{m}
\end{aligned}
$$

Therefore, from (90),

$$
\begin{aligned}
|y(t)| \geq \bar{\delta}_{m} & \Longrightarrow \quad \dot{V}_{0}(t) \leq-\left(k_{2} \bar{\delta}_{m}-k_{1} \delta_{M}-|\varrho(t)|\right)|y| \\
& \Longrightarrow \quad \dot{V}_{0}(t) \leq-c_{\varrho} \sqrt{2 V_{0}}
\end{aligned}
$$

where the last inequality comes from (44). We deduce from this inequality that

1. If $\left|y\left(t_{1}\right)\right| \leq \bar{\delta}_{m}$ then $|y(t)| \leq \bar{\delta}_{m}$ for any $t \geq t_{1}$;

2. If $\left|y\left(t_{1}\right)\right|>\bar{\delta}_{m}$ then, by application of the comparison lemma to the above inequality, $|y(t)| \leq \bar{\delta}_{m}$ for any $t \geq$ $t_{1}+\frac{\left|y\left(t_{1}\right)\right|-\bar{\delta}_{m}}{c_{\varrho}}$.

Then, it follows from (91) that $|y(t)| \leq \bar{\delta}_{m}$ for any $t \geq T(y(0)):=$ $t_{1}+\max \left\{0, \frac{|y(0)|+\left(k_{1} \delta_{M}+|\varrho|_{s}\right) t_{1}-\bar{\delta}_{m}}{c_{\varrho}}\right\}$. In other words, $\overline{\operatorname{sat}}_{\bar{\delta}}(y(t))=y(t)$ for $t \geq T(y(0))$.

Proof of Lemma 3: It relies on the following result (Hua et al., 2009, Prop. 1) 
Proposition 7 Consider a smooth function $\zeta$ with $|\zeta|=1$ and $\dot{\zeta}$ independent of $\omega$. Let $\bar{\zeta}:=R^{T} \zeta$ and

$$
\left\{\begin{array}{l}
\omega_{1}=-k_{4} \frac{\bar{\zeta}_{2}}{\left(1+\bar{\zeta}_{3}\right)^{2}}-\zeta^{T} S\left(R e_{1}\right) \dot{\zeta} \\
\omega_{2}=k_{4} \frac{\bar{\zeta}_{1}}{\left(1+\bar{\zeta}_{3}\right)^{2}}-\zeta^{T} S\left(R e_{2}\right) \dot{\zeta}, \quad k_{4}>0
\end{array}\right.
$$

Then, on the unit sphere, $\operatorname{Re}_{3}=\zeta$ is exponentially stable with convergence domain $\left\{R(0) e_{3}: \zeta^{T}(0) R(0) e_{3} \neq-1\right\}$.

We apply the above proposition with $\zeta:=\frac{R \mu}{|\mu|}$. First, $\zeta$ is well defined because, from (20) and (23), $\mu$ never vanishes. Then, $\zeta$ is a smooth function since both $R$ and $\mu$ are smooth. Let us check that $\dot{\zeta}$ is independent of $\omega$. First, recall that by Def. 1$i), R \operatorname{sat}(z)=\operatorname{sat}(R z)$ for any $R, z$. Therefore, from (20) and the definitions of $\gamma, \tilde{v}$, and $a_{r}$,

$$
\zeta=\frac{g e_{3}+k_{1} \operatorname{sat}_{\delta}(R \eta)+k_{2} \overline{\operatorname{sat}}_{\bar{\delta}}(\tilde{p})-\ddot{p}_{r}}{\left|g e_{3}+k_{1} \operatorname{sat}_{\delta}(R \eta)+k_{2} \overline{\operatorname{sat}}_{\bar{\delta}}(\tilde{p})-\ddot{p}_{r}\right|}
$$

The derivatives of $g e_{3}, k_{2} \overline{\operatorname{sat}}_{\delta}(\tilde{p})$ and $\ddot{p}_{r}$ do not depend on $\omega$. As for $R \eta$, it follows from (6) and (18) that $\frac{d}{d t}(R \eta)=-k_{3}(R \eta-M \tilde{p})$. This term is thus also independent of $\omega$. Thus $\dot{\zeta}$ is independent of $\omega$.

By replacing $\zeta$ in (92) by the expression $\zeta:=\frac{R \mu}{|\mu|}$, one obtains after a few calculation the expression (19). By application of Prop. 7, we deduce that $R e_{3}=\zeta$ is exponentially stable with convergence domain $\left\{R: \zeta^{T}(0) R(0) e_{3} \neq-1\right\}$. Thus, $\exists c, \alpha>0:\left|\operatorname{Re}_{3}-\zeta\right| \leq c\left|\left(\operatorname{Re}_{3}-\zeta\right)(0)\right| e^{-\alpha t}, \forall t$. This is equivalent to $\left|e_{3}-\frac{\mu}{|\mu|}\right| \leq c\left|\left(e_{3}-\frac{\mu}{|\mu|}\right)(0)\right| e^{-\alpha t}, \forall t$. Since, from A1, (20), and (23), $|\mu|$ is lower and upper-bounded by strictly positive constants independent of the initial conditions, the above inequality is equivalent to $|\tilde{\mu}(t)| \leq \bar{c}|\tilde{\mu}(0)| e^{-\alpha t}$ for some constant $\bar{c}$.

Proof of Lemma 4: Since $\mathcal{A}_{\rho *} \subset \mathcal{A}_{\rho}$ when $\rho *<\rho$, it is sufficient to prove the existence of $\varepsilon_{\rho}>0$ for any $\rho>\rho *$, where $\rho *$ is any strictly positive value. Thus, we assume from now on that

$$
\rho>\bar{\delta}_{m}
$$

Let us first establish a few inequalities. From (25) and (27), $|Y|^{2}+\mu_{3}^{2}=|\mu|^{2}>g^{2} \varkappa^{2}$. Thus,

$$
\forall t \geq 0, \quad \mu_{3}(t)^{2}>g^{2} \varkappa^{2}-|Y(t)|^{2}
$$

From (27) and Assumption A3,

$$
|\mu|<c_{1}:=k_{1} \delta_{M}+k_{2} \bar{\delta}_{M}+2 g
$$

Therefore, $|Y|=\left|\left(\mu_{1}, \mu_{2}\right)^{T}\right|<c_{1}$ and from (76),

$$
|\dot{y}|<c_{2}:=2\left(k_{1} \delta_{M}+k_{2} \bar{\delta}_{M}+g\right)
$$

Recalling that $y=R \tilde{v}$, it follows from (28) and (96) that

$$
\forall t>0, \quad|y(t)|<\rho+c_{2} t
$$

Since $M$ is constant, (76) implies that $\dot{x}=M y$. Recalling, from (2), that $F_{\delta}$ and $\bar{F}_{\bar{\delta}}$ are bounded by $C_{\delta}$, it follows from (76) that

$$
\varepsilon \dot{Y}=-\mu_{3} Y+\varepsilon \omega_{3}(t) Y^{\perp}+\varepsilon\left(\xi_{1}+\xi_{2} y\right)
$$

where $\xi_{1}, \xi_{2}$ are functions bounded by a constant $c_{3}$ independent of $\varepsilon$. Thus, using the triangular inequality,

$$
\frac{d}{d t}|Y|^{2} \leq|Y|^{2}\left(-\frac{2 \mu_{3}}{\varepsilon}+1\right)+c_{3}^{2}\left(1+|y|^{2}\right)
$$

Let $T_{0}$ denote any strictly positive constant. It follows from (97) and (99) that

$$
\forall t \in\left[0, T_{0}\right], \quad \frac{d}{d t}|Y|^{2}(t) \leq|Y|^{2}(t)\left(-\frac{2 \mu_{3}(t)}{\varepsilon}+1\right)+c_{4}
$$

with

$$
c_{4}:=c_{3}^{2}\left(1+\left(\rho+c_{2} T_{0}\right)^{2}\right)
$$

We show that there exists $\varepsilon_{0}>0$ such that, for any $\varepsilon \leq \varepsilon_{0}$ and any initial condition in $\mathcal{A}_{\rho}$,

$$
\left|Y\left(T_{0}\right)\right|<\bar{\varkappa}:=\min \left\{\frac{9\left(k_{2} \bar{\delta}_{m}-k_{1} \delta_{M}\right)}{10}, g \varkappa / \sqrt{2}\right\}
$$

We claim that there exists $\bar{\varepsilon}_{1}>0$ such that, $\forall \varepsilon \leq \bar{\varepsilon}_{1}$,

$$
\forall t \in\left[0, T_{0}\right], \quad|Y(t)|^{2}<g^{2} \varkappa^{2} / 2
$$

Suppose on the contrary that there exists a sequence $\left(\varepsilon_{n}\right)_{n>0}$ converging to zero such that, for any $\varepsilon=\varepsilon_{n}$ there exists a time $\bar{t}_{n} \in\left[0, T_{0}\right]$ such that $\left|Y\left(\bar{t}_{n}\right)\right|^{2} \geq g^{2} \varkappa^{2} / 2$. Since, from (28), $|Y(0)|^{2}<g^{2} \varkappa^{2} / 2$, we can assume without loss of generality that $|Y(t)|^{2} \leq\left|Y\left(\bar{t}_{n}\right)\right|^{2}=g^{2} \varkappa^{2} / 2$ for $t \leq \bar{t}_{n}$. Therefore,

$$
\frac{d}{d t}|Y|^{2}\left(\bar{t}_{n}\right) \geq 0
$$

On the other hand, from (94), the fact that $\mu_{3}(0)>0$ (cf. (28)), and the fact that $|Y(t)|^{2} \leq g^{2} \varkappa^{2} / 2$ for $t \leq \bar{t}_{n}$, we deduce that $\mu_{3}\left(\bar{t}_{n}\right)>g \varkappa / \sqrt{2}$. Thus, we deduce from (100) and (104) that

$$
0 \leq \frac{g^{2} \varkappa^{2}}{2}\left(-\frac{2 \mu_{3}(\bar{t})}{\varepsilon}+1\right)+c_{4}<\frac{g^{2} \varkappa^{2}}{2}\left(-\frac{\sqrt{2} g \varkappa}{\varepsilon}+1\right)+c_{4}
$$

This is impossible if $\varepsilon<\bar{\varepsilon}_{1}:=g^{3} \varkappa^{3} \sqrt{2} /\left(g^{2} \varkappa^{2}+2 c_{4}\right)$, which shows (103) for $\varepsilon \leq \bar{\varepsilon}_{1}$. From (103), (94), and the fact that $\mu_{3}(0)>0$, it follows that $\mu_{3}(\bar{t})>g \varkappa / \sqrt{2}$ for all $t \in\left[0, T_{0}\right]$. In other words, for $\varepsilon \leq \bar{\varepsilon}_{1}$,

$$
\forall t \in\left[0, T_{0}\right], \quad|Y(t)|^{2}<g^{2} \varkappa^{2} / 2, \mu_{3}(t)>g \varkappa / \sqrt{2}
$$

Therefore, from (100),

$$
\forall t \in\left[0, T_{0}\right], \quad \frac{d}{d t}|Y|^{2}(t) \leq|Y|^{2}(t)\left(-\frac{g \varkappa \sqrt{2}}{\varepsilon}+1\right)+c_{4}
$$

Applying the comparison lemma yields

$$
\forall t \in\left[0, T_{0}\right], \quad|Y|^{2}(t) \leq e^{-a t}\left(|Y|^{2}(0)-\frac{c_{4}}{a}\right)+\frac{c_{4}}{a}
$$

with $a=\frac{g \varkappa \sqrt{2}}{\varepsilon}-1$. Since $a$ tends to infinity as $\varepsilon$ tends to zero and $|Y(0)|<g \varkappa / \sqrt{2}$, there exists $\bar{\varepsilon}_{2}$ such that, $\forall \varepsilon \leq \bar{\varepsilon}_{2}$,

$$
e^{-a T_{0}}\left(|Y|^{2}(0)-\frac{c_{4}}{a}\right)+\frac{c_{4}}{a}<\left(\frac{9\left(k_{2} \bar{\delta}_{m}-k_{1} \delta_{M}\right)}{10}\right)^{2}
$$


This inequality, together with (106) and (103) imply (102) for $\varepsilon \leq \varepsilon_{0}:=\min \left\{\bar{\varepsilon}_{1}, \bar{\varepsilon}_{2}\right\}$.

We now assume that

$$
\varepsilon \in\left(0, \varepsilon_{\rho}\right) \text { with } \varepsilon_{\rho}=\min \left\{\varepsilon_{0}, \frac{\sqrt{2} g \varkappa \bar{\varkappa}^{2}}{\bar{\varkappa}^{2}+c_{4}}\right\}
$$

We claim that

$$
\forall t \geq T_{0},\left\{\begin{array}{l}
|y(t)|<\rho+c_{2} T_{0} \\
|Y(t)|<\bar{\varkappa} \\
\mu_{3}(t)>g \varkappa / \sqrt{2}
\end{array}\right.
$$

Since $\varepsilon<\varepsilon_{0}$, it follows from (97), (102), and (105) that the three inequalities in (108) are satisfied at $t=T_{0}$. Suppose by contradiction that there exists $T>T_{0}$ such that at least one of the three inequalities in (108) is not satisfied at $t=T$ and these inequalities are satisfied for any $t \in\left[T_{0}, T\right)$. We distinguish three cases:

Case 1: $|y(T)|=\rho+c_{2} T_{0}$. We claim that

$$
\forall t \in\left[T_{0}, T\right), \quad|y(t)| \leq \max \left\{\bar{\delta}_{m},\left|y\left(T_{0}\right)\right|\right\}
$$

Indeed, from (108) for $t \in\left[T_{0}, T\right),|Y(t)| \leq \bar{\varkappa}<k_{2} \bar{\delta}_{m}-k_{1} \delta_{M}$ for any $t \in\left[T_{0}, T\right]$. Therefore, by (76),

$$
\forall t \in\left[T_{0}, T\right], \quad|y(t)| \geq \bar{\delta}_{m} \Longrightarrow \frac{d}{d t}|y|(t)<0
$$

Thus, if $\max \left\{\bar{\delta}_{m},\left|y\left(T_{0}\right)\right|\right\}=\left|y\left(T_{0}\right)\right|$ then $|y(t)| \leq\left|y\left(T_{0}\right)\right|$ for all $t \in\left[T_{0}, T\right)$, and if $\max \left\{\bar{\delta}_{m},\left|y\left(T_{0}\right)\right|\right\}=\bar{\delta}_{m}$ then $|y(t)| \leq \bar{\delta}_{m}$ for all $t \in\left[T_{0}, T\right)$. This proves (109). By continuity, $|y(T)| \leq$ $\max \left\{\bar{\delta}_{m},\left|y\left(T_{0}\right)\right|\right\}$. This contradicts the assumption $|y(T)|=\rho+$ $c_{2} T_{0}$ since $\left|y\left(T_{0}\right)\right|<\rho+c_{2} T_{0}$ by (97), and $\bar{\delta}_{m}<\rho$ by (93).

Case 2: $|Y(T)|=\bar{\varkappa}$. Since (108) is satisfied for $t \in\left[T_{0}, T\right)$,

$$
\frac{d}{d t}|Y|^{2}(T) \geq 0
$$

It follows from (99), (101), and (108) for $t<T$, that

$$
\begin{aligned}
\frac{d}{d t}|Y|^{2}(T) & \leq|Y|^{2}(T)\left(-\frac{g \varkappa \sqrt{2}}{\varepsilon}+1\right)+c_{4} \\
& \leq \bar{\varkappa}^{2}\left(-\frac{g \varkappa \sqrt{2}}{\varepsilon}+1\right)+c_{4}<0
\end{aligned}
$$

where the last inequality follows from (107). This contradicts (111).

Case 3: $\mu_{3}(T)=g \varkappa / \sqrt{2}$. Since (108) is satisfied for $t<T$, it follows by continuity that $|Y(T)|^{2} \leq g^{2} \varkappa^{2} / 2$. Thus, $\mu_{3}(T)^{2}+$ $|Y(T)|^{2} \leq g^{2} \varkappa^{2}$, which contradicts (94).

This concludes the proof of (108). Lemma 4 follows from (108) and Lemma 2 applied to the second equation in (76) with $\varrho(t)=R(t)\left(Y_{1}(t), Y_{2}(t), 0\right)^{T}$.

Proof of Lemma 5: It is inspired by the proof of Lemma 4. Let us first define some constant numbers. With $c_{2}$ defined by (96) and $V$ given by (46) the Lyapunov function of Prop. 1, let us define

$$
\begin{aligned}
& T_{0}:=\frac{\bar{\delta}_{m}}{2 c_{2}}, \quad c_{5}:=\left(\rho+\frac{\bar{\delta}_{m} T_{0}}{2}+\frac{c_{2} T_{0}^{2}}{2}\right)|M|_{s} \\
& \bar{V}:=\max \left\{|V(x, y)|:|x| \leq c_{5},|y| \leq \bar{\delta}_{m}\right\} \\
& \bar{c}_{5}:=\max \left\{|x|: \inf _{y} V(x, y) \leq 2 \bar{V}\right\} \\
& c_{6}:=\min \left\{\left|\left(y, \operatorname{sat}_{\delta}(x)\right)\right|: V(x, y)=2 \bar{V}\right\} \\
& \bar{\varkappa}:=\min \left\{\frac{9\left(k_{2} \bar{\delta}_{m}-k_{1} \delta_{M}\right)}{10}, \frac{g \varkappa}{\sqrt{2}}, \frac{9}{10} \frac{\beta c_{6}}{2 c}\right\}
\end{aligned}
$$

with $c$ any constant satisfying (68). Note that $\bar{c}_{5}$ is well defined because $V$ is proper. Note also, from the definition of $\bar{V}$ and $\bar{c}_{5}$, that

$$
c_{5} \leq \bar{c}_{5}
$$

Relations (94) to (96) are still valid when $M$ is not constant. As for (97), using (29) it becomes

$$
\forall t>0, \quad|y(t)|<\frac{\bar{\delta}_{m}}{2}+c_{2} t
$$

Due to the term $\dot{M} \tilde{p}=\dot{M} M^{-1} x$ in the expression (76) of $\dot{x}$, relation (98) becomes

$$
\varepsilon \dot{Y}=-\mu_{3} Y+\varepsilon \omega_{3}(t) Y^{\perp}+\varepsilon\left(\xi_{1}+\xi_{2} y+\xi_{3} x\right)
$$

with $\xi_{1}, \xi_{2}, \xi_{3}$ functions bounded by a constant $c_{3}$ independent of $\varepsilon$. Thus, by the triangular inequality (99) becomes

$$
\frac{d}{d t}|Y|^{2} \leq|Y|^{2}\left(-\frac{2 \mu_{3}}{\varepsilon}+1\right)+c_{3}^{2}\left(1+|y|^{2}+|x|^{2}\right)
$$

Since $\dot{\tilde{p}}=R \tilde{v}=y,|\tilde{p}(t)-\tilde{p}(0)| \leq \int_{0}^{t}|y(s)| d s$ for all $t$. Thus, from (29) and (115), $|\tilde{p}(t)|<\rho+\frac{\bar{\delta}_{m} t}{2}+\frac{c_{2} t^{2}}{2}$ for any $t>0$, which implies by the definition (113) of $c_{5}$ that

$$
\forall t \in\left(0, T_{0}\right], \quad|x(t)|<c_{5} \leq \bar{c}_{5}
$$

where the last inequality comes from (114). We deduce from (115), (117), and (118) that (100) is satisfied with $c_{4}$ now defined by

$$
c_{4}:=c_{3}^{2}\left(1+\bar{\delta}_{m}^{2}+\bar{c}_{5}^{2}\right)
$$

From here, one can proceed as in the proof of Lemma 4 to show that for $\varepsilon$ smaller than some $\varepsilon_{0}>0$, (102) and (105) hold true with $\bar{\varkappa}$ defined by (113).

Recall from (113) that $T_{0}:=\frac{\bar{\delta}_{m}}{2 c_{2}}$. Thus, from (115), $\left|y\left(T_{0}\right)\right|<$ $\bar{\delta}_{m}$. We impose on $\varepsilon$ the condition (107), with $c_{4}$ defined by (119). We claim that there exists $\vartheta>0$ such that, provided that $|\dot{M}|_{s} \leq \vartheta$

$$
\forall t \geq T_{0}, \begin{cases}i)|V(x(t), y(t))|<2 \bar{V} & \text { ii) }|y(t)|<\bar{\delta}_{m} \\ i i i)|Y(t)|<\bar{\varkappa} & \text { iv) } \mu_{3}(t)>\frac{g \varkappa}{\sqrt{2}}\end{cases}
$$

The four properties in (120) are satisfied at $t=T_{0}$. Indeed, by (113), (115), and (118), $\left|V\left(x\left(T_{0}\right), y\left(T_{0}\right)\right)\right| \leq \bar{V}$. Property ii), at $t=T_{0}$, follows from (115). Properties iii-iv) follow from (102) and (105), which are satisfied because $\varepsilon<\varepsilon_{0}$. To prove (120) we proceed by contradiction. Suppose that for any $\vartheta>0$ there exists some $M$ with $|\dot{M}|_{s} \leq \vartheta$ and some $T>T_{0}$ such that at least one of the properties in (108) is not satisfied at $t=T$ and these properties are satisfied $\forall t \in\left[T_{0}, T\right)$. We distinguish four cases. Case 1: $|V(x(T), y(T))|=2 \bar{V}$. Since (120) is satisfied for $t \in$ $\left[T_{0}, T\right)$, one has

$$
\dot{V}(x(T), y(T)) \geq 0
$$

One also deduces from $(120)$ on the time-interval $\left[T_{0}, T\right)$ that on the time-interval $\left[T_{0}, T\right]$, the function $\overline{\mathrm{sat}}_{\bar{\delta}}$ desaturates. Thus, from (76), the solution satisfies on $\left[T_{0}, T\right]$ :

$$
\left\{\begin{array}{l}
\dot{x}=M y+\dot{M} \tilde{p} \\
\dot{y}=-k_{1} \operatorname{sat}_{\delta}(x)-k_{2} y+R\left(Y_{1}, Y_{2}, 0\right)^{T}
\end{array}\right.
$$


This system corresponds to (54) modulo the additive "perturbation" $R\left(Y_{1}, Y_{2}, 0\right)^{T}$. It follows from (120) for $t \in\left[T_{0}, T\right]$ that $|V(x(T), y(T))| \leq 2 \bar{V}$. From (113), this implies that $|x(t)| \leq$ $\bar{c}_{5} \forall t \in\left[T_{0}, T\right]$. Using the same argument as for (59), one deduces that $\forall t \in\left[T_{0}, T\right],|x(t)| \leq \frac{\left|\mathrm{sat}_{\delta}(x)\right|}{s_{m}}$ with $s_{m}:=\min _{|x| \leq \bar{c}_{5}} \mathrm{~s}_{\delta}\left(|x|^{2}\right)$. With this definition of $s_{m}$, it follows from (60) that the derivative of $V$ along the solutions of (122) satisfies, on the time-interval $\left[T_{0}, T\right]$ :

$$
\begin{aligned}
\dot{V} & \leq-C_{2,1}|y|^{2}+C_{2,2}|y|\left|\operatorname{sat}_{\delta}(x)\right|-C_{2,3}\left|\operatorname{sat}_{\delta}(x)\right|^{2} \\
& +\frac{\partial V}{\partial y} R\left(Y_{1}, Y_{2}, 0\right)^{T}
\end{aligned}
$$

with $C_{2,1}, C_{2,2}, C_{2,3}$ defined by (61). From (61), the coefficients $C_{2, j}$ tend to $C_{1, j}$ as $\vartheta$ tends to zero. Therefore, by (53) there exists $\vartheta>0$ such that, for $|\dot{M}|_{s} \leq \vartheta$,

$$
\dot{V} \leq-\frac{\beta}{2}\left(|y|^{2}+\left|\operatorname{sat}_{\delta}(x)\right|^{2}\right)+\frac{\partial V}{\partial y} R\left(Y_{1}, Y_{2}, 0\right)^{T}
$$

Using (68), we then deduce that

$$
\dot{V} \leq-\frac{\beta}{2}\left(|y|^{2}+\left|\operatorname{sat}_{\delta}(x)\right|^{2}\right)+c\left|\left(y, \operatorname{sat}_{\delta}(x)\right) \| Y\right|
$$

It thus follows from (120) for $t \in\left[T_{0}, T\right)$ that, on the timeinterval $\left[T_{0}, T\right]$,

$$
\dot{V} \leq-\frac{\beta}{2}\left(|y|^{2}+\left|\operatorname{sat}_{\delta}(x)\right|^{2}\right)+c \bar{\varkappa}\left|\left(y, \operatorname{sat}_{\delta}(x)\right)\right|
$$

From (113) and the assumption $V(x(T), y(T))=2 \bar{V}$, one deduces that $\mid\left(y(T), \operatorname{sat}_{\delta}(x(T)) \mid \geq c_{6}\right.$. Then, it follows from (126) and (113) that $\dot{V}(x(T), y(T))<0$. This contradicts (121).

Case 2: $|y(T)|=\bar{\delta}_{m}$. Since (120) is satisfied for $t \in\left[T_{0}, T\right)$, it follows that $\frac{d}{d t}|y|^{2}(T) \geq 0$. Since $|Y(t)| \leq \bar{\varkappa}$ on $\left[T_{0}, T\right]$, relation (110) still holds, which implies that $\frac{d}{d t}|y|^{2}(T)<0$, thus a contradiction.

Case 3: $|Y(T)|=\bar{\varkappa}$. Since (120) is satisfied for $t \in\left[T_{0}, T\right)$, it follows that $\frac{d}{d t}|Y|^{2}(T) \geq 0$. Furthermore, (120) for $t<T$ and (113) imply that $x(T) \leq \bar{c}_{5}$. This implies, from (117) and (120) for $t<T$, that (112) is satisfied with $c_{4}$ defined by (119). We thus obtain a contradiction.

Case 4: $\mu_{3}(T)=g \varkappa / \sqrt{2}$. One proceeds as in Case 3 of Lemma 4 to show that this case also yields a contradiction.

We thus have proved that (120) is satisfied if $\vartheta>0$ is chosen small enough. Property a) of Lemma 5 follows from $i$ ) in (120) and (113). Properties b-c) correspond to ii-iii) in (120). Finally, since $|x(t)| \leq \bar{c}_{5}$, (125) is satisfied, which corresponds to Property d) of Lemma 5.

\section{References}

Abdessameud, A., Tayebi, A., 2010. Global trajectory tracking control of vtoluavs without linear velocity measurements. Automatica 46, 1053-1059.

Aguiar, A. P., Hespanha, J. P., Pascoal, A. M., 2007. Switched seesaw control for the stabilization of underactuated vehicles. Automatica 43 (12), 1997 2008.

Aguiar, A. P., Pascoal, A. M., 2007. Dynamic positioning and way-point tracking of underactuated auvs in the presence of ocean currents. International Journal of Control 80 (7), 1092-1108.

Brescianini, D., D'Andrea, R., 2016. Design, modeling and control of an omnidirectional aerial vehicle. In: IEEE Conf. on Robotics and Automation.
Caballero, F., Merino, L., Ferruz, J., Ollero, A., 2009. Vision-based odometry and slam for medium and high altitude flying uavs. Journal of Intelligent and Robotic Systems 54 (1-3), 137-161.

Chen, M., Desoer, C., 1982. Necessary and sufficient condition for robust stability of linear distributed feedback systems. International Journal of Control 35, 255-267.

Conte, G., Doherty, P., 2008. An integrated uav navigation system based on aerial image matching. In: IEEE Aero. Conf. pp. 1-10.

Cunha, R., Silvestre, C., Hespanha, J., Aguiar, A. P., 2011. Vision-based control for rigid body stabilization. Automatica 47 (5).

de Plinval, H., Morin, P., Mouyon, P., 2012. Nonlinear control of underactuated vehicles with uncertain position measurements and application to visual servoing. In: American Control Conference.

Doyle, J., Francis, B., Tannenbaum, A., 1992. Feedback Control Theory. Dover.

Doyle, J. C., Stein, G., 1981. Multivariable feedback design: Concepts for a classical/modern synthesis. IEEE Trans. on Auto. Control 26, 4-16.

Hauser, J., Sastry, S., Meyer, G., 1992. Nonlinear control design for slightly non-minimum phase systems: Application to v/stol. Automatica 28, 651670.

He, Y., Chen, B. M., Wu, C., 2005. Composite nonlinear control with state and measurement feedback for general multivariable systems with input saturation. Systems \& Control Letters 54, 455-469.

Hua, M., Hamel, T., Morin, P., Samson, C., 2009. A control approach for thrustpropelled underactuated vehicles and its application to vtol drones. IEEE Trans. on Automatic Control 54, 1837-1853.

Isidori, A., Marconi, L., Serrani, A., 2003. Robust autonomous guidance : an internal model approach. Advances in industrial control. Springer Verlag.

Khalil, H., 2002. Nonlinear systems, 3rd Edition. Prentice Hall.

Le Bras, F., Hamel, T., Mahony, R., Treil, A., 2010. Output feedback observation and control for visual servoing of vtol uavs. International Journal of Robust and Nonlinear Control 21, 1-23.

Lòpez-Araujo, D., Zavala-Rio, A., Fantoni, I., Salazar, S., Lozano, R., 2010. Global stabilisation of the pvtol aircraft with lateral force coupling and bounded inputs. Int. J. of Control 83, 1427-1441.

Lupashin, S., Schoellig, A., Sherback, M., D'Andrea, R., 2010. A simple learning strategy for high-speed quadrocopter multi-flips. In: IEEE Int. Conf. on Robotics and Automation. pp. 1642-1648.

Ma, Y., Soatto, S., Kosecka, J., Sastry, S., 2003. An Invitation to 3-D Vision: From Images to Geometric Models. SpringerVerlag.

Mahony, R. E., Kumar, V., Corke, P., 2012. Multirotor aerial vehicles: Modeling, estimation, and control of quadrotor. IEEE Robotics and Automation Magazine 19 (3), 20-32.

Marconi, L., Naldi, R., 2007. Robust full degree-of-freedom tracking control of a helicopter. Automatica 43 (11), 1909 - 1920.

Masselli, A., Zell, A., 2012. A novel marker based tracking method for position and attitude control of mavs. In: Int. Micro Air Vehicle Conference and Flight Competition. pp. 1-6.

Mellinger, D., Michael, N., Kumar, V., 2012. Trajectory generation and control for precise aggressive maneuvers with quadrotors. The International Journal of Robotics Research, 1-11.

Metni, N., Hamel, T., Derkx, F., 2004. A uav for bridges inspection: Visual servoing control law with orientation limits. In: 5th Symposium on Intelligent Autonomous Vehicles (IAV 04).

Mondragon, I. F., Campoy, P., Martinez, C., Olivares-Mendez, M., et al., 2010. $3 \mathrm{~d}$ pose estimation based on planar object tracking for uavs control. In: IEEE Conf. on Robotics and Automation.

Pebrianti, D., Kendoul, F., Azrad, S., Wang, W., Nonami, K., 2010. Autonomous hovering and landing of a quad-rotor micro aerial vehicle by means of on ground stereo vision system. Journal of System Design and Dynamics 4, 269-284.

Pflimlin, J.-M., Souères, P., Hamel, T., 2007. Position control of a ducted fan vtol uav in crosswind. Int. J. of Control 80, 666-683.

Saripalli, S., Montgomery, J. F., Sukhatme, G. S., 2003. Visually guided landing of an unmanned aerial vehicle. Robotics and Automation, IEEE Transactions on 19 (3), 371-380.

Teel, A. R., 1991. Global stabilization and restricted tracking for multiple integrators with bounded controls. Systems \& Control Letters 18, 165-171.

Teel, A. R., 1996. A nonlinear small gain theorem for the analysis of control systems with saturation. IEEE Trans. on Automatic Control 41, 1256-1270. 